\title{
Mixing Models and Frame Moduli Bounds \\ In Complex Lithologies
}

\begin{abstract}
A Thesis
Presented to

the Faculty of the Department of Earth and Atmospheric Sciences

University of Houston
\end{abstract}

In Partial Fulfillment

of the Requirements for the Degree

Master of Science

By

Aslan Gassiyev

December 2008 


\section{Mixing Models and Frame Moduli Bounds \\ In Complex Lithologies}

Aslan Gassiyev

APPROVED:

Dr. John Casey, Chairman

Dr. John Castagna, Advisor

Dr. Evgeni Chesnokov, Committee Member

Dr. Tad Smith, Committee Member

Dean, College of Natural Sciences and Mathematics 


\title{
Mixing Models and Frame Moduli Bounds \\ In Complex Lithologies
}

\begin{abstract}
An Abstract of a Thesis
Presented to

the Faculty of the Department of Earth and Atmospheric Sciences

University of Houston
\end{abstract}

In Partial Fulfillment

of the Requirements for the Degree

Master of Science

By

Aslan Gassiyev

December 2008 


\begin{abstract}
In this thesis, I investigated the use of bounding equations in complex porous multiminerallic lithologies to determine bounds for frame elastic moduli by considering different scenarios for porosity distribution. Two different schemes for selection of endmember components are considered. Porosity-explicit mixing model considers each of the mineral constituents and the pore fluid as end-members of the mixture. This approach requires the mixing of dissimilar constituents such as solids and fluids, resulting into elastic moduli bounds, generally, too wide to be useful in porous composites. Alternatively, porosity-implicit mixing model incorporates the porosity into single-phase end-member components. In this case, the elastic properties of porous rocks of differing composition are relatively similar and the corresponding bounds are much tighter than those obtained using porosity-explicit mixing.
\end{abstract}

For a 5\% evenly distributed porosity of a quartz-calcite mixture, Hashin-Shtrikman bounds $\left(K_{H S}\right)$ derived from porosity-implicit mixing model are very close when assumes a linear relationship between compressional velocity and composition: $K_{H S_{+}}-K_{H S_{-}} \approx 0-$ $0.22 \mathrm{GPa}$. However, by correlating compressional velocity to the $3 / 2$ power of calcite, the bounds are much closer. On the other hand, porosity-explicit mixing model yields very wide bounds, e.g. $K_{H S+}-K_{H S-} \approx 43.9-67 G P a$. Approximately, porosity-implicit mixing model yields $96.5-100 \%$ tighter bounds than porosity-explicit mixing model. 
Initially, the model investigated here is restricted to quartz and calcite minerals with both zero and evenly distributed porosity. These results are compared to empirical results of Wilkens et al (1984). Subsequently, effect of uneven porosity distribution between endmembers is added to the model. Comparison of results shows that porosity distribution is an important aspect that needs to be taken into consideration. As our model shows, if we assume an incorrect porosity distribution, the error on $K_{H S+}$ and $K_{H S-\text {, with changing }}$ porosity $\varphi=1-13 \%$, will be from $1.2 \%$ to around $19.1 \%$ and from $2.3 \%$ to around $25.7 \%$ respectively. Results also show that uneven porosity distribution yields tighter bounds than even distribution, e.g. at $\varphi=7 \% K_{H S}$ bounds with uneven distribution are $32.7 \%$ tighter than $K_{H S}$ bounds with even porosity distribution. The results presented here are theoretically exact for dry rocks. 


\section{Contents}

1. Introduction 1

2. Theoretical background 6

2.1 Porosity-Explicit Mixtures 6

2.2 Porosity-Implicit Mixtures $\quad 10$

$\begin{array}{ll}2.3 \text { Theoretical Bounds for Composites } & 14\end{array}$

2.4 Hashin-Shtrikman (1963) Bounding Equations 18

3. Application of Bounds to Dry Quartz-Calcite Mixtures 22

3.1 Form of the Regression Equation $\quad 22$

3.2 Bounds for Zero Porosity Quartz-Calcite Mixtures 25

3.3 Porosity-Explicit Bounds for Dry Porous Quartz-Calcite Mixtures 33

3.4 Porosity-Implicit Bounds for Dry Porous Quartz-Calcite Mixtures 39

4. Discussion of Bounding Equations for Composite Media 41

4.1 Effect of Distribution of Porosity between End-Members on Bounds 43

5. Comparison to Observation 62

6. Conclusions and Discussion 69

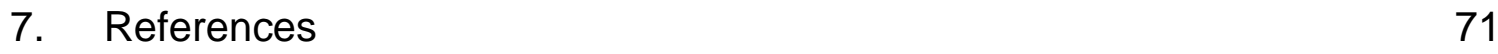




\section{Introduction}

Seismic velocities depend on the lithology, fluid type and content of the formation: therefore, it is necessary to have accurate and exact information about relationships between elastic moduli or velocities and porosity, lithology, and fluid type.

Empirical relationships between the compressional wave velocity $V p$ and shear wave velocity $V s$ in pure lithologies are commonly used in exploration geophysics. In this thesis work, I investigate the prediction of shear-wave velocity given compressional wave velocity and composition in mixed lithologies. To calculate compressional and shear wave velocities we need to know bulk modulus $K$, shear modulus $\mu$, and density $\rho$, in order to apply them to widely used formulas shown below:

$$
v_{p}=\sqrt{\frac{k+\frac{4}{3} \mu}{\rho}} \quad v_{s}=\sqrt{\frac{\mu}{\rho}}
$$

It is much easier to measure compressional wave velocity $V p$, rather than shear wave velocity Vs. Compressional wave velocities are usually measured using logging tools, seismic, but shear wave velocities are often not measured and must be estimated. Commonly, $V s$ is estimated from empirical $V p-V s$ relationships, (e. g., Greenberg and Castagna, 1992). In mixed lithologies, simple interpolations between predictions for pure lithologies are applied. Here, I will investigate the use of bounding equations to constrain predictions in mixed lithologies.

To usefully predict seismic velocities from rock properties alone we need to know details of the microstructure. However, if $\mathrm{P}-$ wave velocity and composition are known, 
empirically it was proved (Greenberg and Castagna, 1992) that useful predictions of Swaves can be made without explicit knowledge of microstructure. Thus, by applying theoretical bounding equations like Reuss-Voigt and the tighter Hashin-Shtrikman bounds we should be able to theoretically explain empirical predictions of $\mathrm{S}$-waves, and even make better estimates of shear wave velocities without direct measurements in mixed lithologies. Basically, to accomplish this we need to know the elastic moduli of a mixed lithology rocks. As will be shown, porosity distribution is a key assumption for elastic moduli prediction.

Composite medium mixing models predict, or place bounds on, the properties of mixtures (i.e., aggregates) of rock-forming constituents. In general, without knowing details of the microstructure, it is not possible to usefully predict seismic velocities from compositional data and theory alone. When applied directly to mixtures of pure constituents, such as minerals and fluids, bounding equations (such as the well known Reuss-Voigt bounds and the tighter Hashin-Shtrikman bounds) are generally too wide in porous rocks to be used in a practical way for seismic lithologic determination. But again, it has been shown empirically (e. g., Greenberg and Castagna; 1992) that if P-wave velocity and composition are known, useful predictions of shear-wave velocity can be made. This suggests that an alternative view of theoretical bounding equations could be found to explain the success of such methods, and to perhaps discover theoretically approaches that make better estimates of shear-wave velocities without direct measurements in mixed lithologies. 
Specifically, the problem of predicting the elastic properties of a mixed-lithology rock given its mineral composition and porosity is addressed in this thesis. A porous rock is a mixture and may be viewed as a composite medium consisting of an aggregate of minerals (including cements) and pore-filling fluid. The elastic properties of the composite depend not only on the properties and proportions of the constituents, but on the structural/geometrical arrangement of those constituents. Composite medium theory relates the properties of the composite to the properties, proportion, and arrangement (including shape) of the constituents (Berryman, 1995). Theories commonly used in geophysics include sphere-pack models (e.g., Gassmann, 1951) and inclusion models (e.g., Kuster and Toksoz; 1974). In general, these theories over-simplify the actual arrangement of constituents and, though very useful for developing physical insight into the systematics of relationships, they cannot generally be used to make predictions in a quantitative sense without combination with empirical equations (e.g., $\mathrm{Xu}$ and White, 1995). Methods considering complex structural arrangements, such as finite-element simulation (Lamb et al.; 1992), suffer from our general inability to adequately characterize the microstructural arrangement needed to simulate the elastic properties of a given rock (see for example Hadley; 1976). Thus, methods of predicting composite properties that do not rely on specific knowledge of the structural arrangement of constituents are needed; models of this type require only the properties and proportions of the individual constituents (which are treated as end-members of the mixture). Such mixing models may be (1) empirical in origin; based on statistical analysis of laboratory and/or well-log information (such as the well known time-average equation; Wyllie et al., 
1956), (2) theoretical, in that even in the absence of precise structural information theoretical bounds can be established, or (3) some combination of the two (e.g., Greenberg and Castagna, 1992). As a first step, the compositional system investigated here is restricted to two minerals, quartz and calcite. This work will also demonstrate the effect of porosity distribution between end-members on the Hashin-Shtrikman bounds. Results of calculations made for rocks assuming equal distribution of porosity will be compared with those made on rock of the same mineral composition, but possessing unequal distribution of porosity.

This thesis will investigate the use of bounding equations in complex porous multiminerallic lithologies to constrain and guide empirical prediction of rock frame moduli and shear-wave velocity given $\mathrm{P}$-wave velocity and composition, and to determine bounds on frame elastic moduli given the elastic moduli of pure end-member lithologies, with particular emphasis on the choice of end-member components. In particular, assuming that mineral composition and porosity are known, two different schemes for selection of end-member components will be considered. The first, porosity-explicit mixture modeling is the traditional approach that treats the pore-fluid as a distinct component, whereas the second, porosity-implicit mixture modeling incorporates the pore-filling fluid into the properties of the porous monominerallic endmembers. Following Berryman and Milton (1991), the end-members are themselves porous and there is no explicit treatment of porosity as a constituent. Porosity-explicit and porosity-implicit models have significantly different bounds. When compared to laboratory measurements in a suite of low porosity rocks consisting primarily of quartz 
and calcite of various proportions, it is found that selection of porosity-implicit endmembers (1) results in convergence of bounds thereby allowing relatively precise prediction of frame moduli given end-member moduli, (2) defines the form of empirical equations that can be used to fit the non-linear compositional dependence of velocityporosity relationships, and (3) results in improved prediction of shear-wave velocities given only compressional-wave velocities and mineral composition.

Predictions made using both approaches will be compared to the empirical linear regression trends reported by Wilkens et al. (1984) in a suite of low porosity rocks containing primarily quartz and calcite as mineral constituents. 


\section{Theoretical Background}

\subsection{Porosity-Explicit Mixtures}

For properties, such as density, for which there is a linear relationship between the property of the composite and the properties of the constituents, composite medium modeling can be accomplished with simple linear mixing equations. In the case of density, the simple mass-balance equation is theoretically exact:

$$
\rho=(1-\phi) \sum_{i=1}^{n} \rho_{i} \chi_{i}+\rho_{f} \phi
$$

where,

$$
\begin{aligned}
& \rho=\text { rock bulk density } \\
& \phi=\text { total porosity, } \\
& \rho_{f}=\text { density of pore-filling fluid, } \\
& \chi_{i}=\text { fraction of total mineral volume occupied by mineral constituent } i, \text { and } \\
& n=\text { number of mineral constituents. }
\end{aligned}
$$

The fractions of total mineral volume are defined such that:

$$
\sum_{i=1}^{n} \chi_{i}=1
$$

If the pore-filling fluid is also a mixture, then the total fluid density can similarly be determined by mixing the fluid phases by a volume weighted linear average of the densities of the individual fluid phases. 
If porosity is treated explicitly as the fraction of total rock volume occupied by porefilling fluid and the mineral constituent volume fractions are expressed as fractions of total rock volume then:

$$
\sum_{i=1}^{n+1} X_{i}=1
$$

where, $X i$ are the end-member volume fractions each for each mineral phase and the fluid, and the number of end-members is now one more than the number of mineral constituents (if the fluid is handled as a single phase). Then, from equation (1):

$$
\rho=\sum_{i=1}^{n+1} \rho_{i} X_{i}
$$

where, $\rho_{f}$ is one of the end-members densities, $\rho_{i}$, and $\phi$ is one of the $X_{i}$.

Other rock properties that are linear, or are nearly linear with composition or porosity over some range, may be treated in an analogous fashion. For example, the slowness (reciprocal velocity) of compressional waves can sometimes be expressed in terms of constituent slownesses and volume fractions by a generalization of the time average equation (Wyllie at al., 1956) that is commonly used in well log analysis:

$$
\Delta t_{p}=\sum_{i=1}^{n+1} \Delta t_{i} X_{i}
$$

where

$\Delta t_{p}=$ bulk rock compressional-wave reciprocal velocity (transit-time), $\Delta t_{i}=$ end-member compressional-wave reciprocal velocity 
In this case, the volume fraction of the fluid end-member is equal to the porosity, and the reciprocal velocity of the fluid end-member (the effective fluid transit-time) is one of the $\Delta t_{i}$.

Although there is no theoretical basis for equation (3) for waves with wavelengths longer than the rock pores and grains, multiple linear regression of measured reciprocal velocity versus end-member volume fractions often yields regression coefficients that are close to the actual mineral and fluid reciprocal velocities (see for example Schlumberger log analysis chart books prior to 1980). Thus, the Wyllie equation (3) can be viewed as a mixing model that explicitly treats porosity as an end-member component.

Similarly, various authors (Tosaya, 1982; Wilkens et al., 1984; Castagna et al., 1985; Han et al., 1986) present linear velocity-composition-porosity relationships for well-lithified rocks that are in form algebraically equivalent to:

$$
V=\sum_{i=1}^{n+1} V_{i} X_{i}
$$

where, $V$ is compressional-or shear-wave velocity of the bulk rock and $V_{i}$ are apparent velocities of the mineral and fluid components (although the pore fluid may be a mixture of several fluid phases it will be treated in this thesis as a single phase with the properties of the fluid mixture). Once again, the volume fraction of the pore fluid is taken to be equal to the porosity. In practice, the apparent velocity of the dominant mineralogical constituent is usually close to the actual mineral velocity, whereas apparent velocities of minor constituents and the pore fluid may have little relationship to actual velocities 
(which in the extreme case of shear-wave velocity for fluids should be zero). There is no theoretical basis for equation (4) and the apparent velocities, $V_{i}$, are most properly thought of as being simply empirical coefficients. However, equation (4) does constitute a commonly employed porosity-explicit mixing model and it will be instructive to compare this model to bounding equations of various kinds as well as to a porosityimplicit mixing scheme discussed below.

It is important to note that the apparent fluid velocity or reciprocal velocity is independent of mineral composition for all porosity-explicit models. Thus, for a porosity-explicit model to adequately describe the behavior of a composite medium, the variation of velocity with porosity must be independent of mineral composition. Clearly, we would expect different porosity dependence in a vuggy limestone than for granular sandstone. This is a fundamental inadequacy of the porosity-explicit approach. 


\subsection{Porosity-Implicit Mixtures}

Berryman and Milton (1991) discuss a composite medium (illustrated in Figure 1) where a porous rock with two mineral phases and one fluid phase (a porosity-explicit ternary mixture) is considered a binary mixture of two porous end-member lithologies (a porosity-implicit binary mixture).

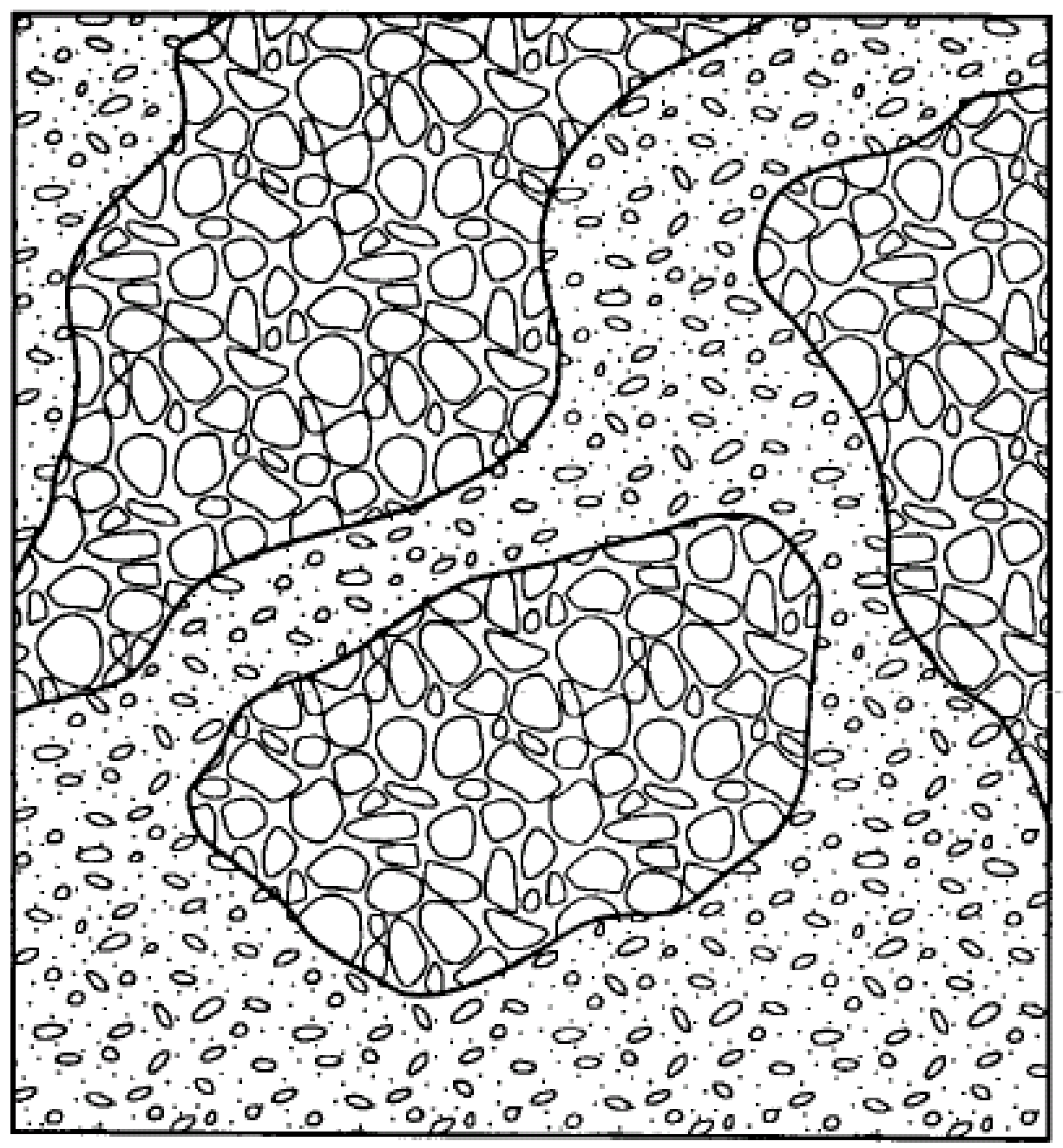

Figure 1: Two types of porous components (end-members) completely fill the volume of composite porous material (from Berryman and Milton, 1991). 
We can generalize this view of mixtures to $n$ porous end-member components whereby the explicit use of porosity as a constituent is avoided. In this porosity-implicit approach, the end-members or phases are porous monomineralic rocks; the effects of the pore-fluid being implicitly contained in the properties of the porous end-members. The number of end-member components is equal to the number of mineralogical components, $n$, and the fractions of total rock volume, $x_{i}$, of the porous end-members sum to unity. The resulting mixing models are analogous to those for porosity-explicit mixing with the exception that the pore-filling fluid is not explicitly considered. Thus, for linear velocity mixing the analogy to equation (4) is:

$$
V=\sum_{i=1}^{n} V_{i} x_{i}
$$

where, the $V_{i}$ represent velocities of the monomineralic porous end-member components rather than mineral or fluid constituent properties. The velocities of the mineralogically pure components can be expressed as any applicable velocity-porosity transform, such as the Hicks (1956) linear velocity-porosity relation:

$$
V_{i}=V_{m i}+c_{i} \phi
$$

where, $V_{m i}$ are the velocities of the zero-porosity mineral constituents, and $c_{i}$ are empirical porosity coefficients. In contrast to the porosity-explicit approach, where there is the same porosity dependence for all compositions, it is apparent in equation (6) that the velocity dependence on porosity is now dependent on composition. There is also now an additional degree of freedom for each additional mineralogical constituent as compared to the porosity-explicit approach, so one must be careful to test the statistical 
significance of resulting regression coefficients used to model observed velocity given porosity and composition data. Practical experience suggests that statistical significance cannot always be achieved and that the additional degrees of freedom resulting from a porosity-implicit approach are not always justified by the data. In this case, the $c_{i}$ can be set equal. On the other hand, it should be intuitively obvious that if pore structure is lithology related, one should expect different velocity-porosity dependencies in lithologies that exhibit very different pore-type compressibilities.

The use of equation (6) in a porosity-implicit mixing model is essentially a first order approximation, whereby the porosity is assumed to be the same in each end-member. This may not necessarily be an adequate representation of reality. For example, consider a porous limestone with isolated quartz grain inclusions, where the porosity exists almost entirely within the limestone end-member.

A more general application of porosity-implicit mixing allows the porosity to differ for the different end-members (Berryman, 1995) in which case equation (6) can be generalized to:

$$
V_{i}=V_{m i}+c_{i} \phi_{i},
$$

where, the $\phi_{i}$ are the porosities of each end-member lithology. This adds additional degrees of freedom to any empirical fit to observations; thus, it is easier to model observed behavior with equation (7) yet more difficult to achieve statistically significant regression fits. It is generally good practice to first attempt to fit the observations with as 
few coefficients as possible, and then to add parameters in a stepwise manner based on physical reasoning that is then substantiated by statistical significance tests.

The general porosity-implicit model reduces to a porosity-explicit mixture when the component porosities are taken to be zero for all end-members with the exception of a fluid-phase end-member which is assigned a porosity of $100 \%$.

A more rigorous theoretical analysis is needed before such simple empirical mixing models can be employed with confidence. In particular, we would like to know if linear compositional dependence as expressed in equation (5) can be justified theoretically, or is at least consistent with theoretical bounding equations. 


\subsection{Theoretical Bounds for Composites}

Let us assume that we are dealing with a macroscopically homogeneous composite of randomly oriented mineral constituents. Given the elastic moduli of the mineral constituents it is possible to calculate the moduli of the composite if certain assumptions are made. For example, Voigt (1928) calculated the composite moduli by averaging over all crystal lattice orientations and assuming that the strain is the same throughout the composite when a given stress is applied externally. Reuss (1929) did the same assuming that the stress is internally everywhere the same for a given overall strain of the composite. Hill (1952) proved that these moduli constitute upper (Voigt) and lower (Reuss) bounds providing the assumption of macroscopic homogeneity is statistically valid. While the individual minerals may be anisotropic, their random orientation results in the aggregate composite being isotropic. Thus, it is common to speak of single mineral bulk and shear moduli even when the mineral crystal is anisotropic; in such cases it should be understood that the mineral moduli actually represent those for a zeroporosity composite of randomly oriented mineral grains. Defining the component moduli in this fashion, we can simply express the Voigt and Reuss bounds for an isotropic composite.

Voigt and Reuss developed averaging schemes to estimate the elastic constants of monomineralic aggregates. To apply Voigt and Reuss moduli on rocks they assumed separation of the individual rock components i.e. minerals, pore filling material, and their arrangement. In the Voigt model, shown in Figure 2, the strain is the same throughout 
the composite when a given stress is applied externally. The $n$ individual components are assumed to be isotropic, and their elastic properties are described by bulk modulus $k$, and shear modulus $\mu$. The elastic moduli are:

$$
K_{v}=\sum_{i=1}^{n} x_{i} K_{i}, \mu_{v}=\sum_{i=1}^{n} x_{i} \mu_{i}
$$

where, $K_{v}$ is the composite Voigt-average bulk modulus, $K_{i}$ are the component bulk moduli, $x_{i}$ are the component volume fractions, $\mu_{v}$ is the composite Voigt-average shear modulus, and $\mu_{i}$ are the component shear moduli.

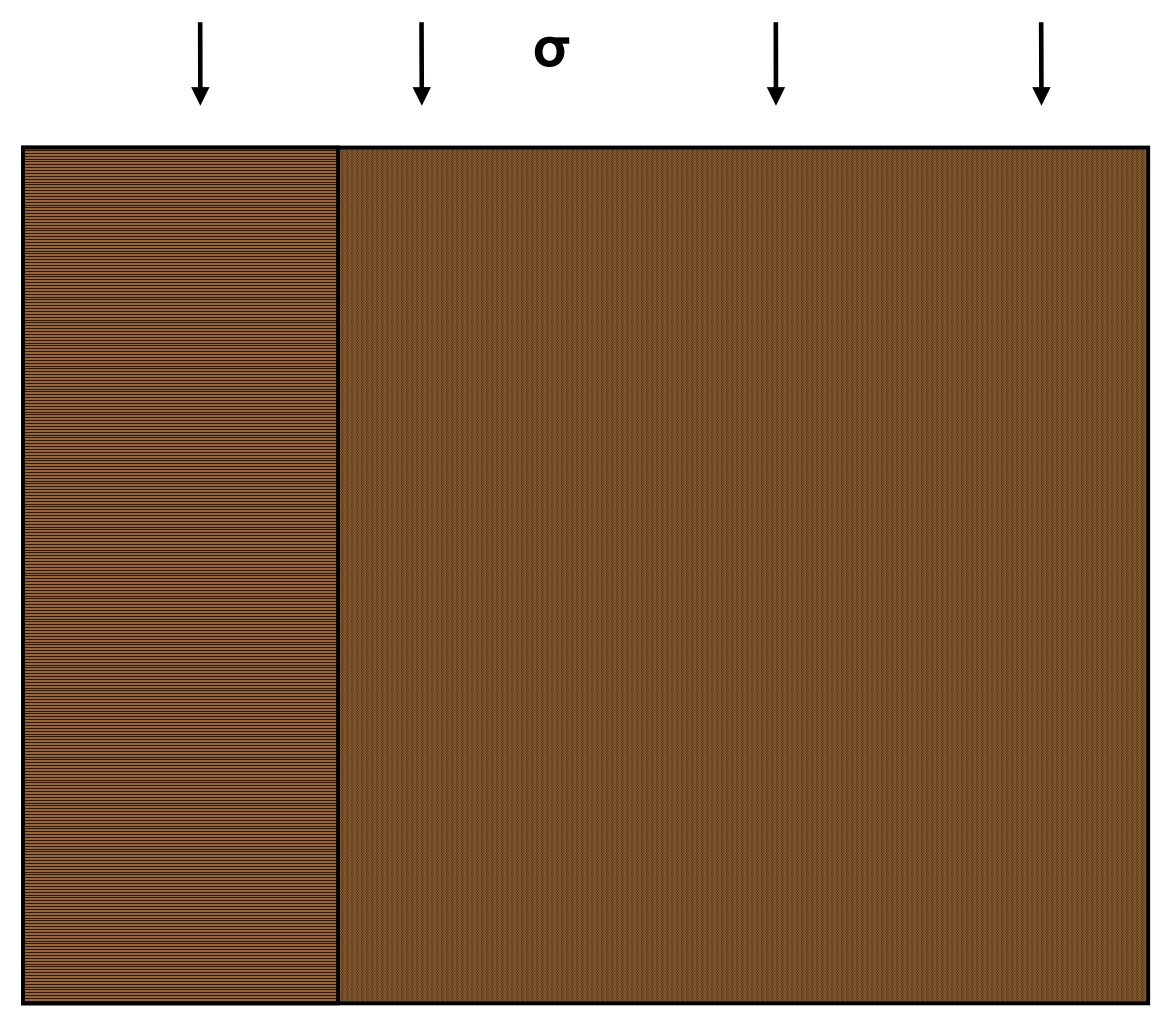

Figure 2. Fundamental model of Voigt (1910) 
In the Reuss model (1927), shown on the Figure 3, the stress is homogeneous and the strain in each layer differs. The elastic moduli are:

$$
1 / K_{r}=\sum_{i=1}^{n} x_{i} / K_{i}, 1 / \mu_{r}=\sum_{i=1}^{n} x_{i} / \mu_{i}
$$

where, $K_{r}$ and $\mu_{i}$ are the composite Reuss-average bulk and shear moduli.

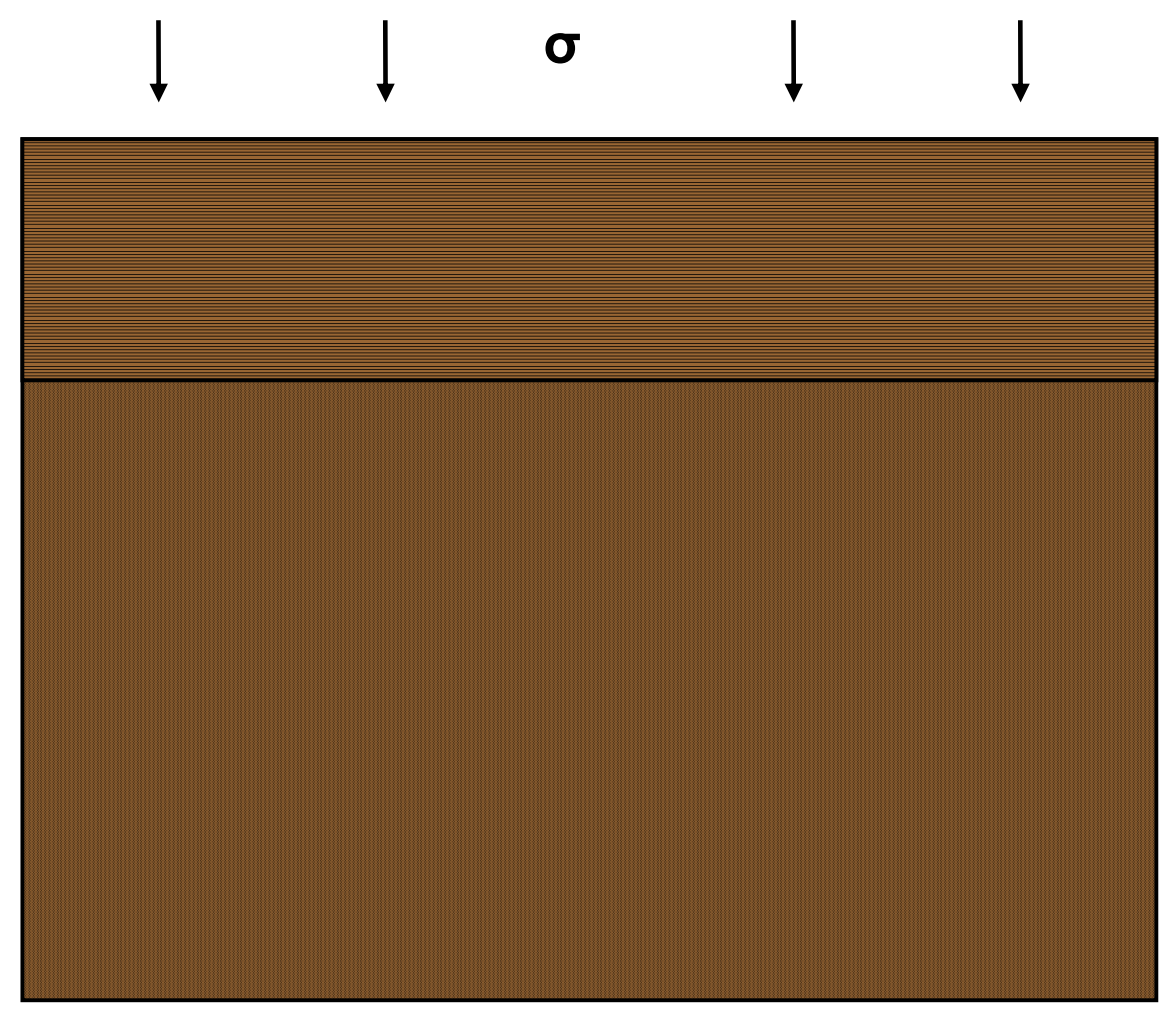

Figure 3. Fundamental model of Reuss (1927)

If we have isotropic effective material, the Voigt and Reuss bulk and shear moduli form bounds on the possible composite model. Thus, the Voigt relationship leads to the upper, and the Reuss relationship to the lower bound. The real values of elastic moduli are situated between these two bounds. 
When the shear-modulus is the same for all constituents, the composite modulus is given by the simple average of the Voigt and Reuss bounds (Hill, 1963). This is called the Hill average. 


\subsection{Hashin-Shtrikman (1963) Bounding Equations}

The Hashin-Shtrikman bounds (Hashin and Shtrikman, 1963) are the tightest possible bounds achievable without specifying the arrangement or shape of the rock constituents. In their work, Hashin and Shtrikman have used variational principles to find bounds for the bulk and shear moduli of composite material. Hashin and Shtrikman assume that the spatial distribution of each component is isotropic, but they did not specifically consider the geometry of the particles or inclusions. For a two component material, these bounds can be easily visualized and correspond to the case of the rock volume being completely filled with spheres each of which contains two concentric layers of the different components. The spheres span a size range from some maximum to infinitely small such that no voids are left unfilled in the total rock volume. The Hashin-Shtrikman upper bound $(H S+)$ corresponds to the case of the outer shell of each sphere being stiffer than the inner core of the sphere, while the lower bound (HS-) corresponds to the core being stiffer than the outer shell (Figure 4). Hashin and Shtrikman (1963) derive these bounds for multiple components, with the requirement that the bulk and shear moduli of the components have the same order of stiffness. Walpole (1966) later showed that the bounds are more broadly applicable and that the bulk and shear moduli need not to be ordered. 
$\mathrm{HS}+$

Upper bounds

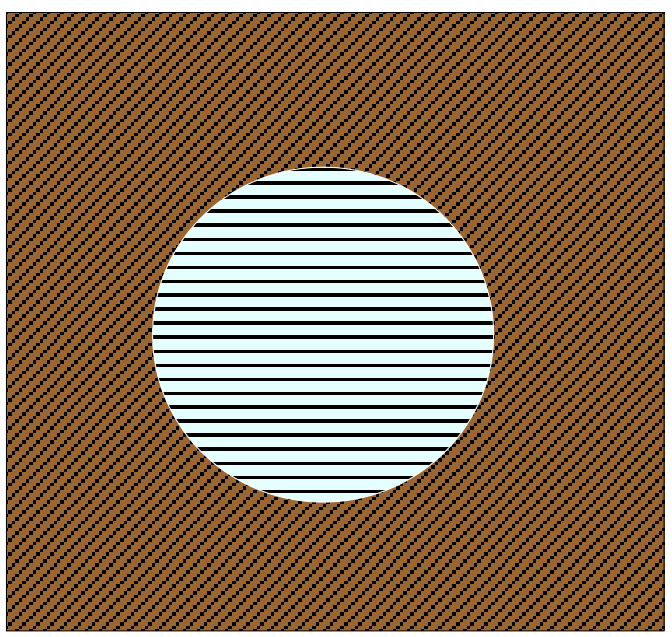

(a)
HS-

Lower bounds

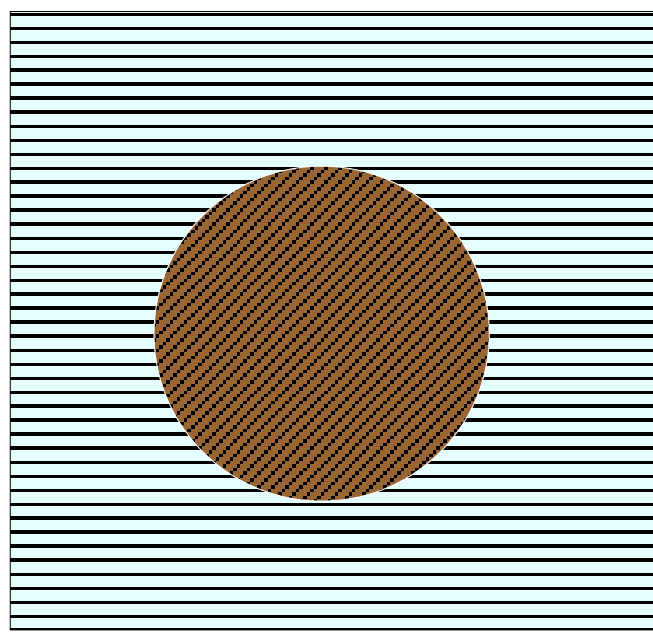

(b)

Figure 4. Fundamental models of Hashin-Shtrikman. (a) Outer shell is stiffer than inner core, which leads to HS+. (b) Inner core is stiffer than outer shell leading to HS-.

The Hashin-Shtrikman bounding equations for bulk modulus are:

$$
K_{H S-}=\left[\sum_{i=1}^{n} \frac{x_{i}}{K_{i}+\frac{4}{3} \mu_{\min }}\right]^{-1}-\frac{4}{3} \mu_{\min }, \quad K_{H S+}=\left[\sum_{i=1}^{n} \frac{x_{i}}{K_{i}+\frac{4}{3} \mu_{\max }}\right]^{-1}-\frac{4}{3} \mu_{\max },
$$


The Hashin-Shtrikman bounding equations for shear modulus are:

$$
\begin{gathered}
\mu_{H S-}=\left[\sum_{i=1}^{n} \frac{x_{i}}{\mu_{i}+\frac{\mu_{\min }}{6}\left(\frac{9 K_{\min }+8 \mu_{\min }}{K_{\min }+2 \mu_{\min }}\right)}\right]^{-1}-\frac{\mu_{\min }}{6}\left(\frac{9 K_{\min }+8 \mu_{\min }}{K_{\min }+2 \mu_{\min }}\right), \\
\mu_{H S+}=\left[\sum_{i=1}^{n} \frac{x_{i}}{\mu_{i}+\frac{\mu_{\max }}{6}\left(\frac{9 K_{\max }+8 \mu_{\max }}{K_{\max }+2 \mu_{\max }}\right)}\right]^{-1}-\frac{\mu_{\max }\left(\frac{9 K_{\max }+8 \mu_{\max }}{K_{\max }+2 \mu_{\max }}\right) .}{}
\end{gathered}
$$

where:

$K_{\min }$ and $K_{\max }$ are the minimum and maximum bulk moduli of the components;

$K_{H S-}$ and $K_{H S+}$ are the Hashin-Shtrikman lower and upper bounds for bulk modulus of the composite;

$\mu_{\min }$ and $\mu_{\max }$ are the minimum and maximum shear moduli of the components;

$\mu_{H S-}$ and $\mu_{H S+}$ are the Hashin-Shtrikman lower and upper bounds for shear modulus of the composite.

When the pore fluid is explicitly treated as a component, the minimum shear modulus, $\mu_{\min }$, is zero, and it is apparent from equations shown above that the HashinShtrikman lower bound for bulk modulus of the composite $\left(K_{H S}\right)$ reduces to the Reuss 
bound for bulk modulus $\left(K_{r}\right)$, while the Hashin-Shtrikman lower bound for the shear modulus $\left(\mu_{H S_{-}}\right)$reduces to the Reuss bound for the shear modulus $\left(\mu_{r}\right)$ and is zero.

Consequently, for porosity-explicit models, even the Hashin-Shtrikman bounds allow a wide range of composite bulk and shear moduli and are consequently not very useful for direct practical application. This can be appreciated by considering tight sandstone, with less than $1 \%$ porosity, where experience dictates that the shear modulus will be a relatively large fraction of the shear modulus of pure quartz, whereas the HashinShtrikman bounds allow a shear modulus of zero. Clearly, a porosity-explicit approach to the bounding equations is inconsistent with our physical insight in this case, and provides little guidance as to how to mix the moduli of end-member lithologies.

In general, for bounds of any kind, the more similar the end-member properties, the more linear the compositional dependence of the composite property, and the tighter the bounds. Conversely, when end-member properties are very dissimilar there is a greater likelihood for highly non-linear behavior with wide bounds. 


\section{Application of Bounds to Dry Quartz-Calcite Mixture}

Wilkens et al. (1984) measured compressional and shear-wave velocities in a suite of siliceous limestones from southeastern Oklahoma as a function of pressure for primarily dry samples, and presented regression trends relating velocities to composition and porosity. In this section, these data are reinterpreted in the context of the mixing models and bounding equations discussed in Section 2.

\subsection{Form of the Regression Equation}

The regressions performed by Wilkens et al. (1984) at each pressure for both compressional and shear-waves were of the form:

$$
V=V_{q t z}+\frac{\partial V}{\partial \chi_{c a l}} \chi_{c a l}+\frac{\partial V}{\partial \phi} \phi,
$$

where, $V_{q t z}$ is the velocity of quartz and $\chi_{\text {cal }}$ is the fraction of total mineral volume of calcite defined so that

$$
\chi_{\text {cal }}=1-\chi_{q t z}
$$

where, $\chi_{q t z}$ is the fraction of total mineral volume of quartz. Given that the volume fractions of calcite and quartz are perfectly negatively correlated as they are defined, the partial derivative must be interpreted in the sense that porosity is maintained constant while the volume fraction of quartz is not. Evaluating (8) for pure zero-porosity calcite gives 


$$
V_{c a l}=V_{q t z}+\frac{\partial V}{\partial \chi_{c a l}},
$$

where $V_{c a l}$ is the resulting velocity of calcite extrapolated by regression to zero porosity.

Since, $X_{i}=(1-\phi) \chi_{i}$, then

$$
\frac{\partial V}{\partial \chi_{c a l}} \chi_{c a l}=\frac{\partial V}{\partial X_{c a l}} X_{c a l}
$$

It can be shown using this identity that equation (8) is algebraically equivalent to equation (4). Writing (4) for three constituents (quartz, calcite, and fluid) gives:

$$
V=V_{q t z} X_{q t z}+V_{c a l} X_{c a l}+V_{\text {fluid }} X_{\text {fluid }}
$$

where $X_{\text {fluid }}$ is the porosity or fraction of fluid and $V_{\text {fluid }}$ is an empirical constant which can be loosely referred to as the effective fluid velocity given by:

$$
V_{\text {fluid }}=V_{q t z}+\frac{\partial V}{\partial \phi}
$$

Equation (11) is readily recast into a form suitable for interpretation of regression analysis in terms of physical parameters by eliminating the redundant parameter $X_{q t z}$ :

$$
V=V_{q t z}+\left(V_{c a l}-V_{q t z}\right) X_{c a l}+\left(V_{\text {fluid }}-V_{q t z}\right) X_{\text {fluid }}
$$

The mixing described by equations (11) and (13) is that of a porosity-explicit mixing model with mineral and fluid constituents being treated exactly the same way and with velocity linearly related to both composition and porosity. The coefficients of equation (13) are readily interpreted in physical terms but the coefficient for porosity $\left(V_{f l u i d}-V_{q t z}\right)$ 
is forced to be independent of composition, which as previously discussed may not be desirable. Furthermore, when the range of porosities or compositions is small, the regression coefficients may have little physical relation to actual component properties. The Wilkens et al. (1984) dataset has a low porosity and the resulting regressions yield a negative $V_{\text {fluid }}$ for P-waves.

Let us consider the regression coefficients given in Table 1 of Wilkens et al. (1984) for compressional and shear-wave velocities at 0.01 kbar as being representative of dry velocities for porous quartz-calcite mixtures. Table 1 provides the Wilkens et al. (1984) coefficients in a form compatible with equation (11). Although the extrapolated fluid velocities are not physical, the quartz and calcite velocities are reasonably close to experimental measurements (see for example Table 1 in Castagna et al, 1993). It should be noted that the regressions for compressional velocity given by Wilkens include some water-saturated measurements, the assumption being that for very low porosities the velocities are almost insensitive to saturation. It is possible that this contributes to the highly non-physical regression fluid velocity for compressional waves.

Table 1: Empirical constants derived from regression coefficients given by Wilkens et al (1984) for dry porous rocks composed primarily of quartz and calcite at $\mathbf{. 0 1}$ kbar. The units are $\mathrm{km} / \mathrm{sec}$.

\section{Compressional}

$V_{q t z}$

$V_{\text {cal }}$

$V_{\text {fluid }}$
6.05

6.46

$-7.14$
Shear

3.70

3.21

0.51 


\subsection{Bounds for Zero Porosity Quartz-Calcite Mixtures}

Given equation (11) and the regression coefficients in Table 1, one can calculate the implied velocities of zero-porosity quartz-calcite mixtures by setting porosity to zero and varying the composition. Using $\rho_{q t z}=2.65 \mathrm{gm} / \mathrm{cc}, \rho_{c a l}=2.72 \mathrm{gm} / \mathrm{cc}$ (as reported by Wilkens et al.1984), and $\rho_{\text {fluid }} \approx 0$ for air, the density for each composition is given by the mass-balance equation and the elastic moduli are then obtained from the velocities. The resulting relationships between velocities or moduli and composition may then be compared to the Reuss-Voigt and Hashin-Shtrikman bounds with end-member properties for quartz and calcite derived from Table 1. The zero porosity trend for bulk modulus versus composition resulting from the Wilkens et al. (1984) regression coefficients is almost identical to the Voigt bound (see Figure 5a). Without further theoretical analysis this can lead to the incorrect interpretation that the common practice of averaging Reuss and Voigt bounds to determine the properties of a zero porosity aggregate is inadequate for this suite of rocks and that the Voigt bound would be a better predictor than the Hill average.

A physical interpretation of this phenomenon would be that this suite of rocks is so welllithified and the porosity so low (with relatively incompressible pores) that the strain is almost uniform throughout the rock. To the contrary, Figure $5 \mathrm{~b}$ also reveals that for zero-porosity quartz-calcite mixtures (1) the Hashin-Shtrikman upper and lower bounds are very close, (2) the Hill average is a good approximation to the Hashin-Shtrikman result (as the shear modulus is similar for quartz and calcite), and (3) the Wilkens et al. 
(1984) regression trend violates the theoretically exact Hashin-Shtrikman bounds for bulk modulus.

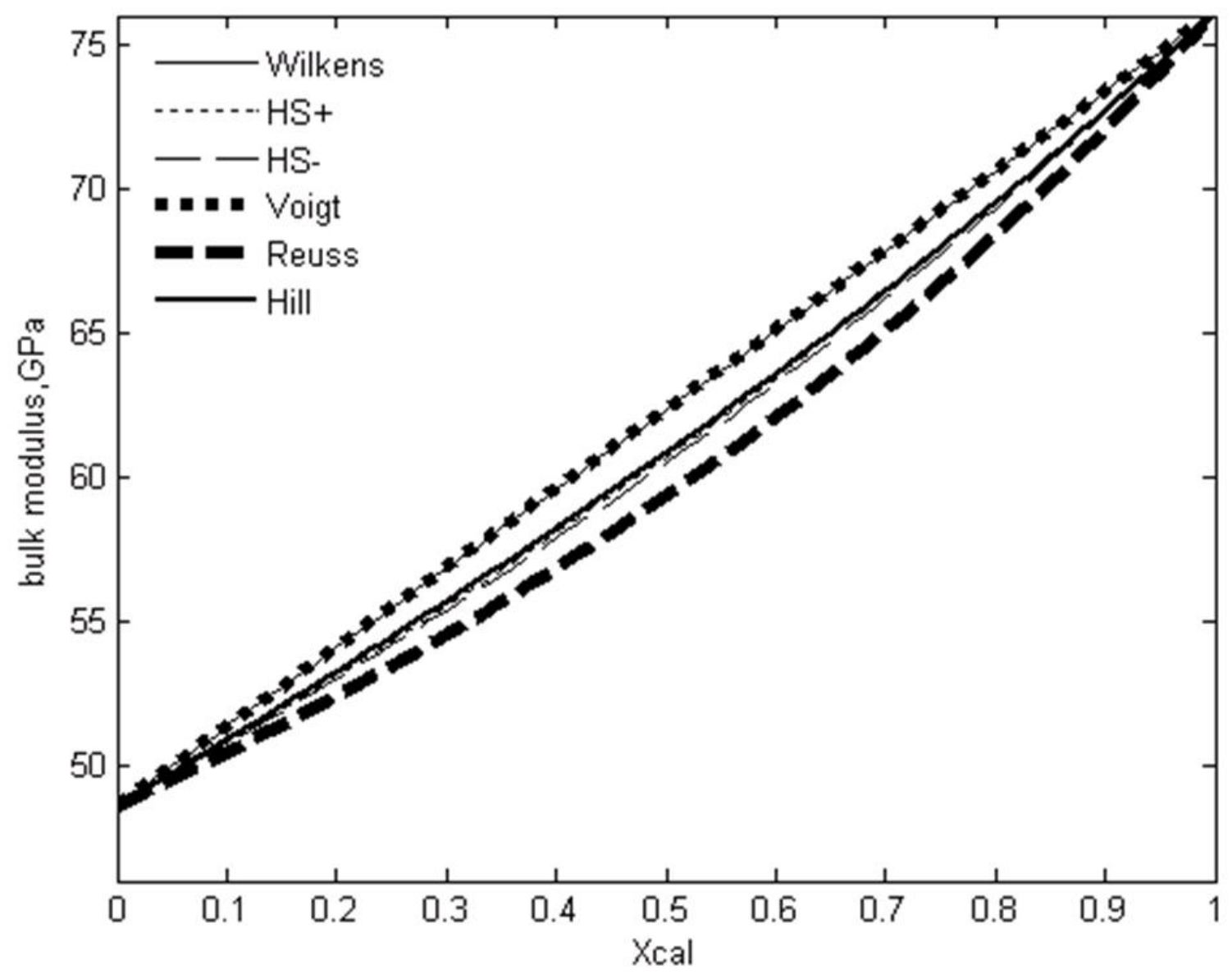

Figure 5a: Bulk modulus versus composition showing Wilkens et al. (1984) regression trend, the Hashin-Shtrikman upper (HS+) and lower (HS-) bounds, the Voigt-Reuss bounds, and the Hill average for a zero-porosity quartz-calcite mixture. The regression trend almost coincides with the Voigt bound; and the Hashin-Shtrikman bounds are almost identical and are well approximated by the Hill average. 


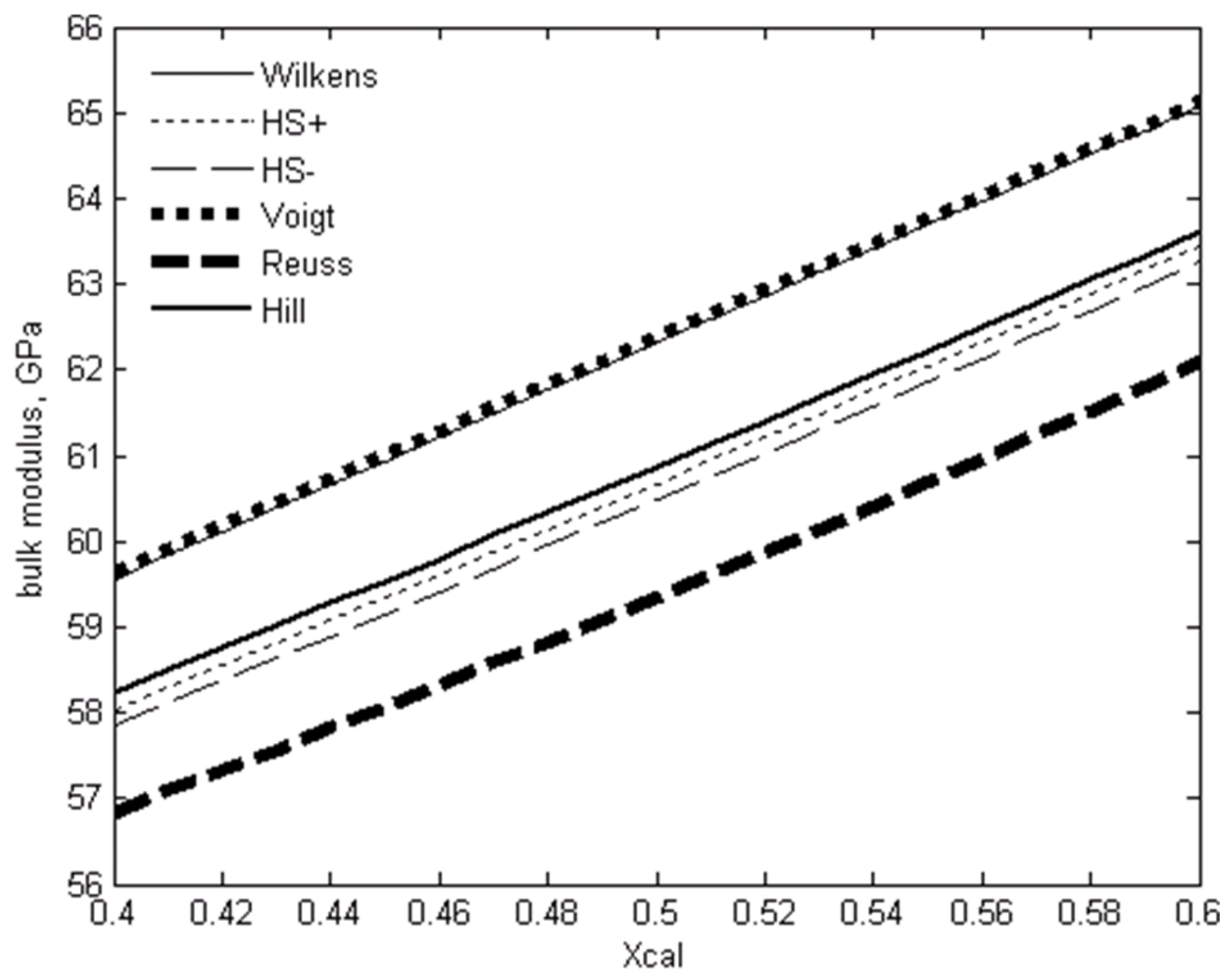

Figure 5b: Zoom of a portion of Figure 2a where the bulk modulus spread is greatest (in the vicinity of equal proportions of constituents). Slight differences between various curves are now apparent. 
The most likely explanation for this discrepancy is that selecting equation (8) as the form used for regression analysis constitutes the arbitrary assumption that velocity is linearly related to composition in the quartz-calcite system; this assumption is apparently unwarranted. Although the regression trend is nearly correct at the compositional extremes of pure quartz or calcite (as evidenced by reasonable agreement with pure mineral measurements) a non-linear relationship between velocity and composition is required by the Hashin-Shtrikman bounds, otherwise it is found that empirical mixing models that are linear in composition violate the porosity-explicit Hashin-Shtrikman bounds.

A similar analysis for the shear modulus (Figure 6a and 6b) results in almost the same conclusions for a zero porosity quartz-calcite mixture. As compared to the bulk modulus results, the regression shear modulus is not quite as close to the Voigt bound, and the Hill average is now within the almost identical Hashin-Shtrikman bounds.

The fact that the Wilkens et al. (1984) regression trend, when extrapolated to zero porosity, violates theoretical bounds for bulk and shear modulus can be due to (1) experimental error, (2) unanticipated fluid effects, (3) inadequacies or misapplication of the theoretical equations, (4) extrapolation of regression trends beyond the range of porosities for which the measurements were made, and/or (5) improper form of the regression trend. There is no reason to suspect that the data or theory is in error. As the suite of rocks investigated by Wilkens et al. (1984) were low porosity it is unlikely that the disagreement is an extrapolation error. Similar discrepancies are also seen for porous 
rocks (as shown below) so another explanation is needed. The form of the regression trend selected by Wilkens et al. (1984) is the simplest form that can be chosen, and is thus the proper choice to use for regression unless there is compelling reason to choose otherwise. This form has been used for most fits to experimental velocity data over a range of compositions (see for example Tosaya, 1982; Castagna et al., 1985; Han et al., 1986; etc.). 


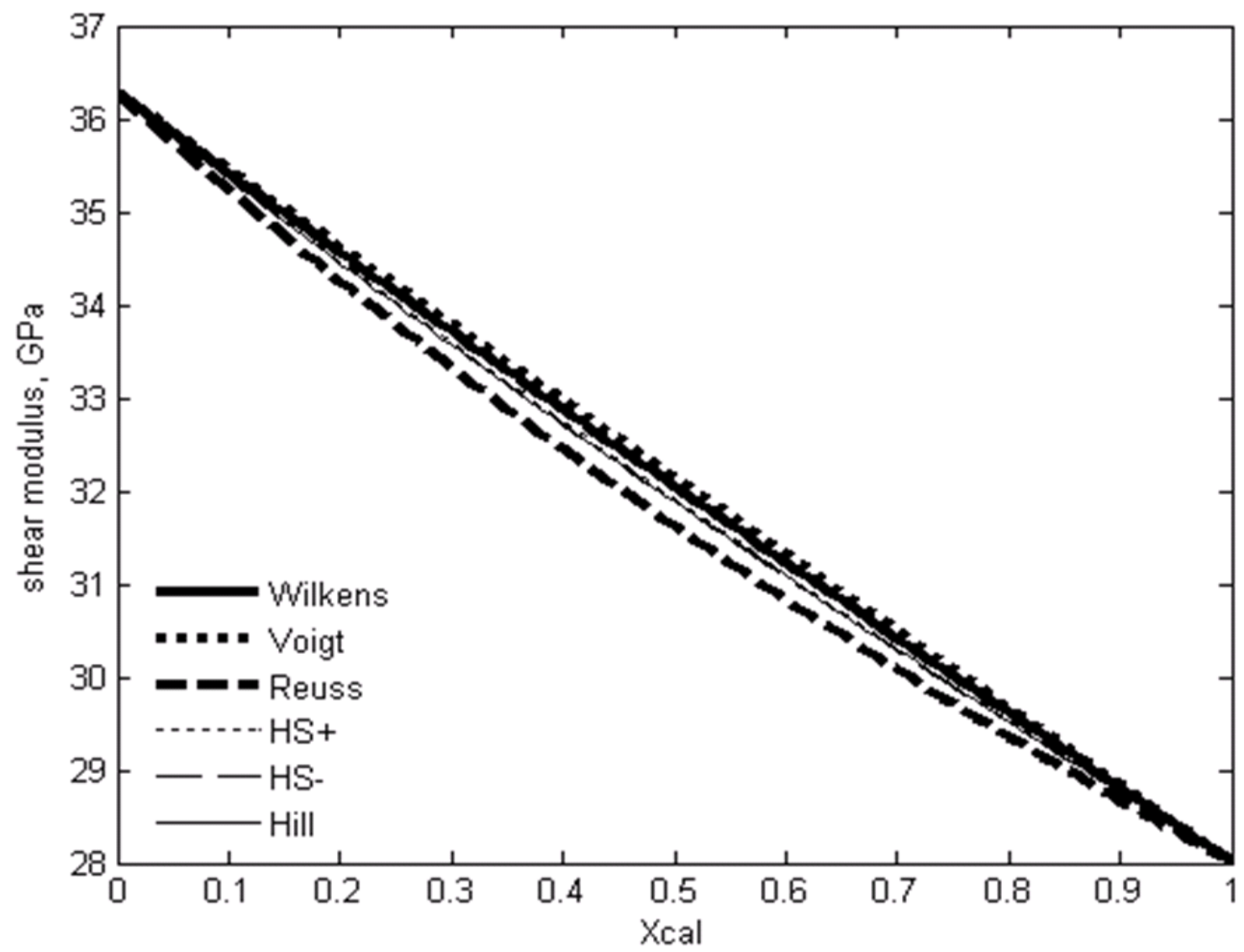

Figure 6a: Shear modulus versus composition showing Wilkens et al. (1984) regression trend, the Hashin-Shtrikman upper (HS+) and lower (HS-) bounds, the Voigt-Reuss bounds, and the Hill average for a zero-porosity quartz-calcite mixture. The regression trend is close to Voigt bound. The Hashin-Shtrikman bounds are almost identical and bound the Hill average. 


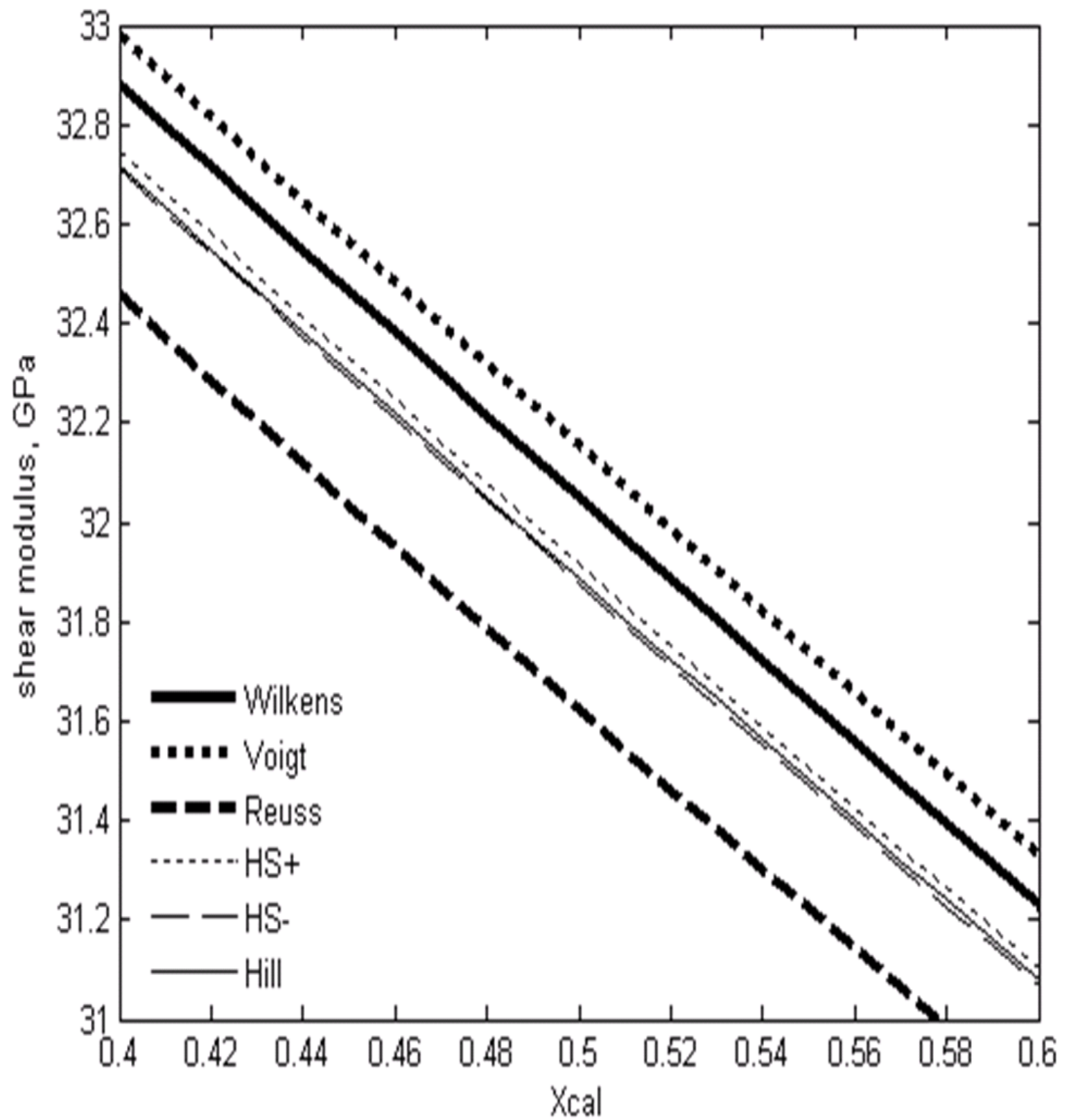

Figure 6b: Zoom of a portion of Figure 6a where the shear modulus spread is greatest (in the vicinity of equal proportions of constituents). Slight differences between various curves are now apparent. 
One could conclude that violation of theoretical bounds is ample reason to consider a regression form that is non-linear in composition if justified by the data. It remains to be seen if the experimental error is so large as to make the non-linearity required by theoretical bounds irrelevant for practical purposes. There were insufficient velocity measurements at zero porosity to directly answer this question for the Wilkens et al. (1984) dataset, so this issue will be considered again below in the context of the full suite of velocity measurements. 


\subsection{Porosity-Explicit Bounds for Dry Porous Quartz-Calcite Mixtures}

Unfortunately, things are not so simple for porous rocks. In contrast to the zero porosity case, where the Hashin-Shtrikman bounds and the Hill average are excellent predictors of mixture properties, these equations are far less helpful in porous rocks when pore filling fluid (such as water or air) is explicitly considered a component of the mixture. If we model the suite of dry rocks investigated by Wilkens et al. (1984) as ternary mixtures of quartz, calcite, and air, at 5\% porosity the bounds are too wide to be useful and the Hill average is not close to the Wilkens et al. (1984) regression trend for bulk and shear moduli (Figures 7 and 8). 


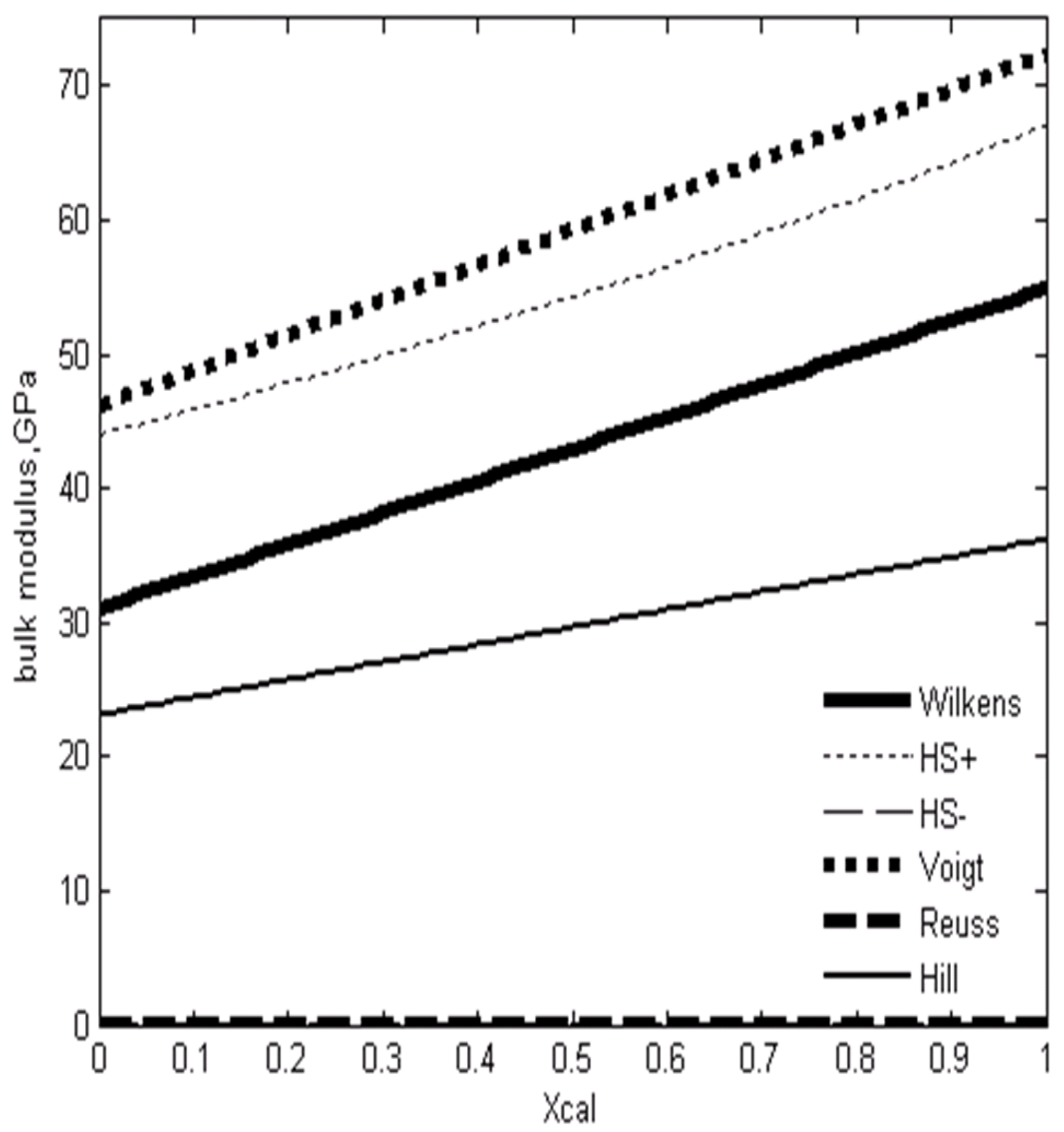

Figure 7: Bulk modulus from Wilkens et al. (1984) regression trends and porosity explicit bounds for 5\% porosity. The Reuss and Hashin-Shtrikman lower bounds are near zero for air-filled pores. 


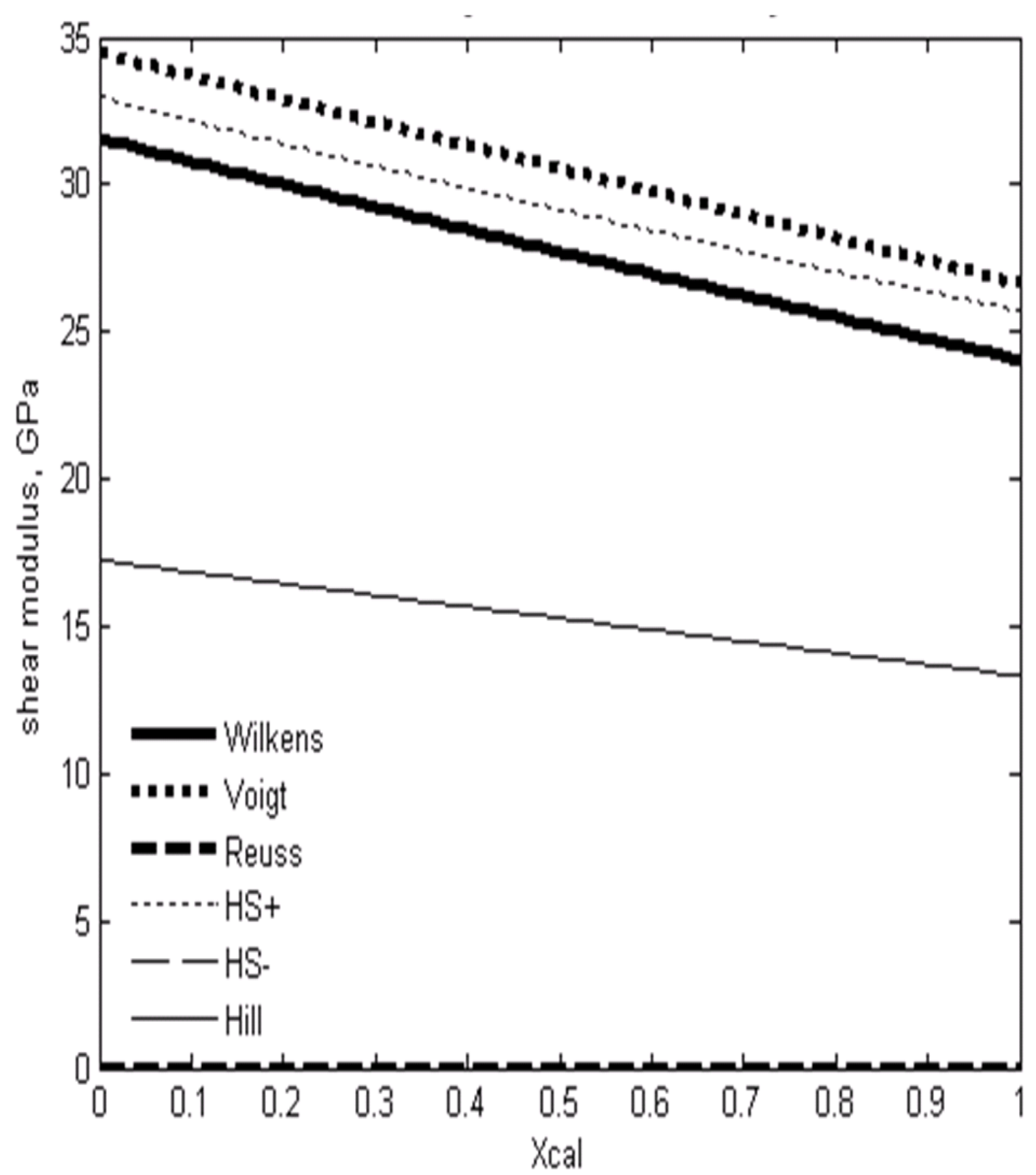

Figure 8: Shear modulus from Wilkens et al. (1984) regression trends and porosity explicit bounds for 5\% porosity. The Reuss and Hashin-Shtrikman lower bounds are zero. 
Also, Figure 9 illustrates that the traditional model of a porous mixture with two minerals (quartz and calcite) is actually a ternary mixture of three end-member components (calcite, quartz and water or air). Mixing equations that deal with porosity explicitly in this way include the Wyllie time-average and Raymer-Hunt-Gardner equations when applied to complex lithologies using an "effective" mineral transit-time or velocity, and the velocity average equations popularized by Tosaya (1982), Wilkens et al., (1984), Castagna et al., (1985) and Han (1986) among others. These equations all deal with the minerals and fluids equally as end-members of the system. In this case, as shown in Figure 10 for a $5 \%$ porosity dry rock, the bounds are too wide to be useful for predictive purposes. Notably, the lower bounds are off the scale (e.g., zero for shear modulus). 


\section{Porosity Explicit Model}

\section{- End-members are minerals and fluid}

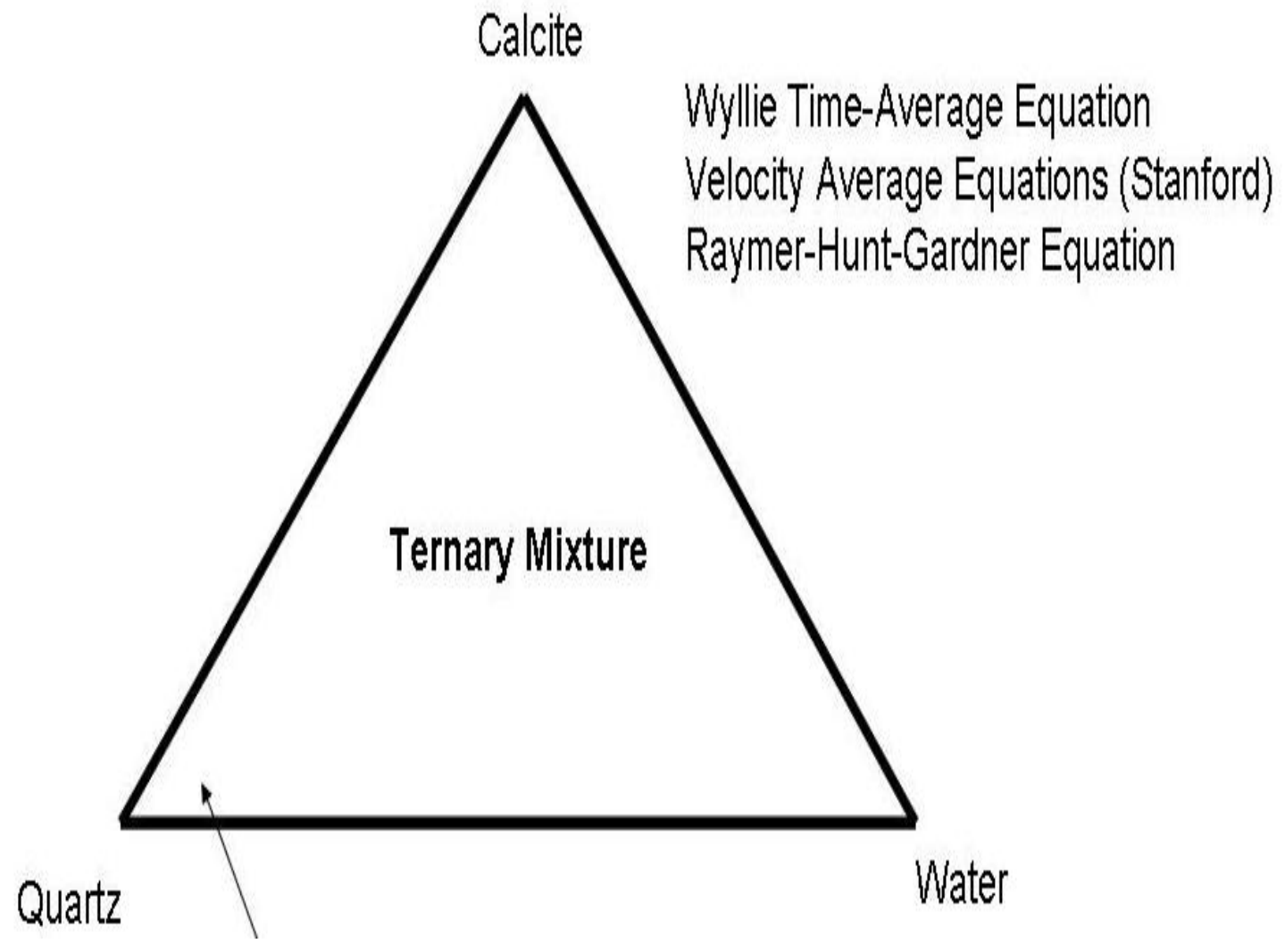

Figure 9: A porosity-explicit representation of a porous quartz-calcite rock as a ternary mixture of quartz, calcite, and fluid such as water. Examples of such models include the Wyllie time-average, the linear velocity average, and the Raymer-HuntGardner equations. 


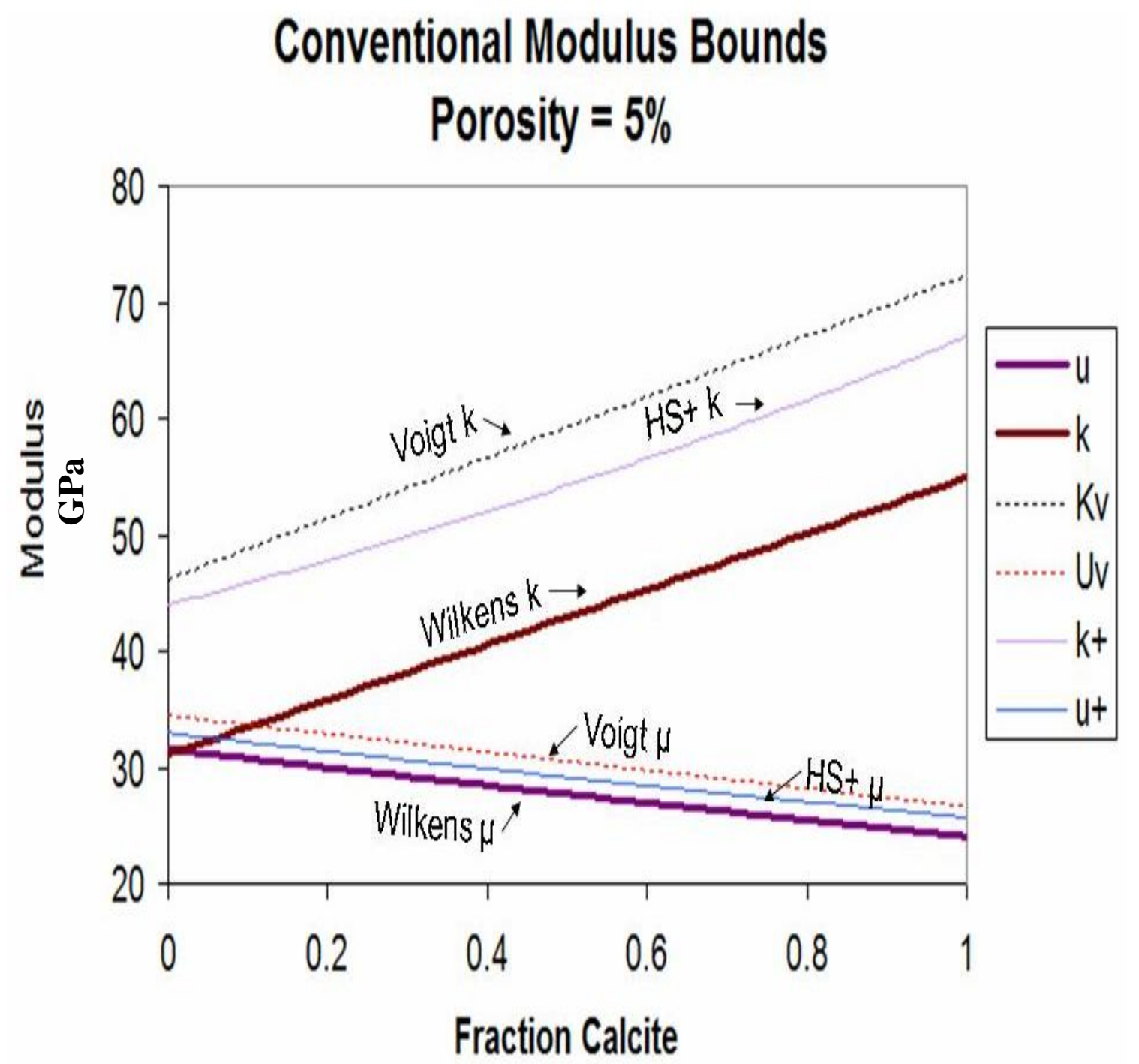

Figure 10: Bulk modulus $(k)$ and shear modulus $(\mu)$ for a $5 \%$ porosity mixture of quartz and calcite. Regression fits by Wilkens et al. (1984) are shown in brown for bulk modulus and purple for shear modulus. The dashed lines are Voigt bounds. The thin solid lines are the Hashin-Shtrikman upper bounds for bulk and shear modulus. The lower bounds are too low to appear on the chart. 


\subsection{Porosity-Implicit Bounds for Dry Porous Quartz-Calcite Mixtures}

In the porosity-implicit approach, explicit ternary mixtures of quartz, calcite, and pore fluid are modeled as binary mixtures of porous quartz and porous calcite. The results depend on how the porosity is apportioned between the end-members. This may be done in a variety of ways:

(1) The simplest way to apportion porosity is to make it the same for both endmembers. This would be a reasonable approach for a granular rock where the porosity is between randomly distributed grains.

(2) The tightest bounds will occur if the porosity is apportioned so as to make the shear modulus equal in both end-members, giving the simple Hill average. Although mathematically simple, this approach is difficult to justify.

(3) The porosity is distributed in the most petrologically reasonable way based on whatever a priori information are available, and/or to best fit the data. Furthermore, the distribution of porosity between end-members could be made dependent on composition.

In this thesis, the first and third approaches will be investigated.

The Wilkens et al. (1984) regression trends are used to calculate the bulk and shear moduli for pure porous sandstone or limestone end-members and the bounding equations are then used to calculate the moduli that result from mixing these lithologies. Figures 10 and 12 show the results, for a total porosity of $5 \%$ that is equally apportioned between sandstone and limestone. It can be seen that the bounds are much tighter for porosityimplicit than for the porosity-explicit case and that relatively precise predictions can be 
made. This is a consequence of the fact that in the porosity-implicit approach, the bulk and shear moduli of the end-members are similar.

The porosity-implicit model suggests a non-linear relationship between $\mathrm{P}$-wave velocity and calcite content that statistically fits the data of Wilkens et al. (1984) better than simple linear regression with the same number of variables. 


\section{Discussion of Bounding Equations for Composite Media}

The Hashin-Shtrikman bounds are tighter than the Reuss-Voigt bounds (Berryman, 1995). The upper Hashin-Shtrikman bounds $(H S+)$ correspond to the case of a stiff matrix within, which soft spherical inclusions exist (an example would be a vuggy limestone). The lower bounds $(H S$-) are the converse; the matrix is soft and the inclusions are stiff (an example would be shale consisting of a small percentage of rounded quartz grains completely encased in a clay "groundmass"). The more or less similar end-member properties yield narrow and semi-linear bounds while very different end-member properties yield wide bounds, which can be highly non-linear with composition. Thus, when porosity is explicitly considered a constituent, bounds for porous rock are usually too wide to be useful. This suggests that, for predictive purposes, it would be most effective to define end-members such that they have the most similar properties possible.

The case of bounds for composites with similar end-member properties is illustrated in Figure 11 for the particular case of bulk and shear moduli for zero porosity quartz-calcite mixtures. Here the Reuss-Voigt and Hashin-Shtrikman bounds are so tight that any reasonable average of the bounds, such as a Hill (1952) average must be similar to actual observations. 


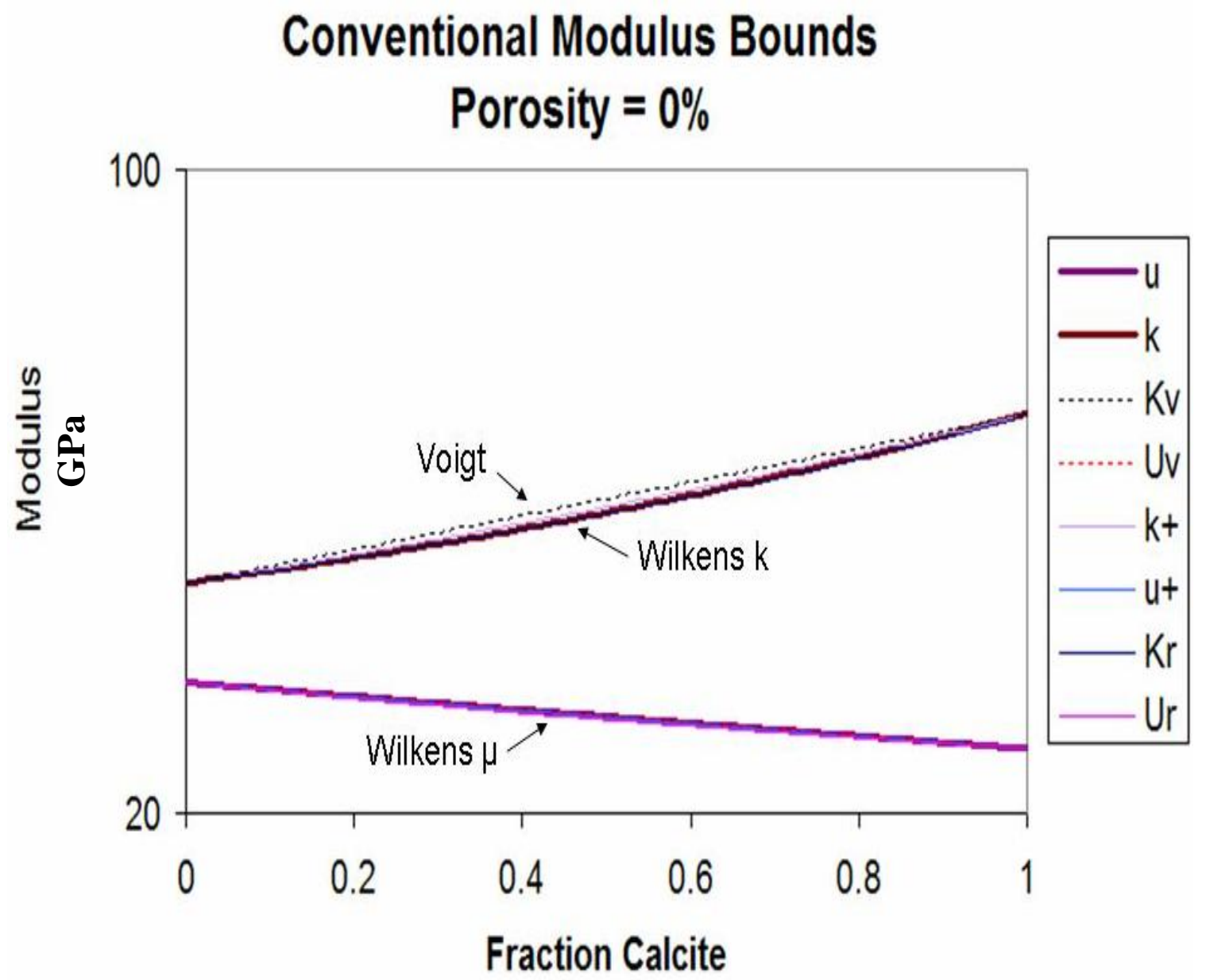

Figure 11: Bulk moduli and shear moduli as a function of composition for a zero porosity mixture of quartz and calcite. Regression fits by Wilkens et al. (1984) are shown in brown for bulk modulus $(k)$ and purple for shear modulus $(\mu)$. The dashed line is the Voigt bound for bulk modulus (Kv). The Reuss bound for bulk modulus (Kr), the Hashin-Shtrikman bounds for bulk (k+) and shear moduli (u+), and the Reuss (Ur) and Voigt (Uv) bounds for shear modulus, all coincide with the observed regression fits. 


\subsection{Effect of Distribution of Porosity between End-Members on the Bounds}

Berryman and Milton (1991) show that for a dry rock frame, it is theoretically exact to avoid dealing explicitly with porosity by incorporating its effect into the end-member properties. In this view of a porous mineral aggregate containing quartz and calcite, we have a binary mixture of porous sandstone and porous limestone. It is relevant how the porosity is distributed between these end-members; as a zeroth order approximation, the porosity is taken to be equally distributed between the end-members to eliminate a degree of freedom in regression fits to data. However, it should be made clear that how porosity is distributed between the end members requires further discussion (see below).

Given empirical trends relating moduli to porosity for pure end-members, we can now use bounds defined in a porosity-implicit way to determine the properties of a mixture components. Figure 12 shows that the Hashin-Shtrikman upper and lower bounds are virtually identical for a $5 \%$ porosity aggregate of quartz and calcite. 


\section{Binary Modulus Bounds}

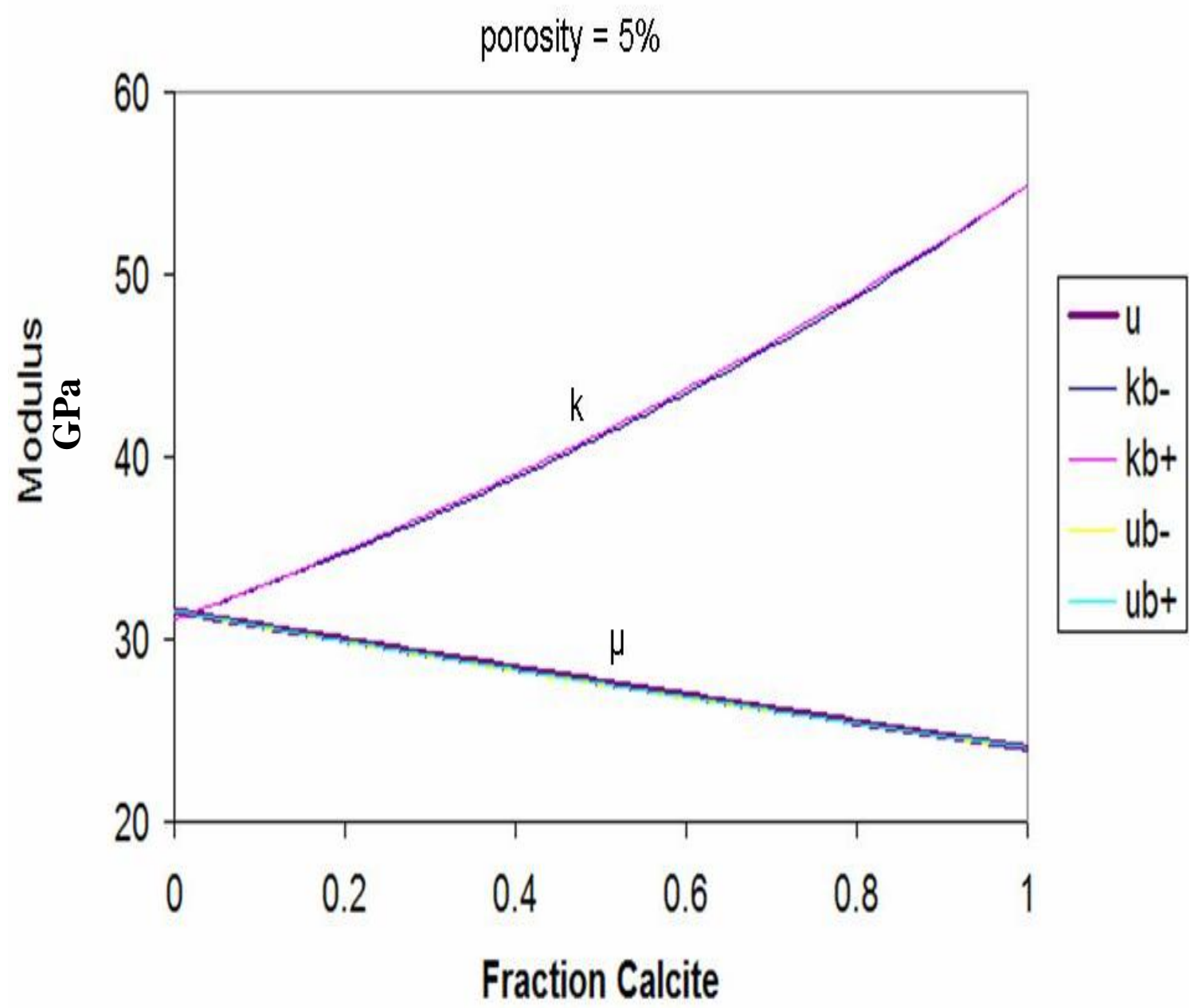

Figure 12: For a binary porosity-implicit mixture, the Hashin-Shtrikman upper and lower bounds are virtually identical for both bulk and shear moduli. The predicted shear modulus is in precise agreement with the linear regression fit (heavy purple line) to the data of Wilkens et al. (1984). 
To illustrate the effects expected at higher porosities it is instructive to consider a binary model with quartz and limestone end-members of equal porosity.

Parameters of this model consist of the fraction of limestone $\left(X_{l m s}\right)$, porosity of limestone $\left(\varphi_{\text {lms }}\right)$, fraction of sandstone $\left(X_{s s}\right)$ that contains $56 \%$ of quartz $\left(X_{q}=56 \%\right)$ and porosity changing: $\varphi=1-24 \%$ :

$$
X_{s s}=X_{q t z}+\varphi_{q t z}
$$

As sandstone porosity increases, composition of limestone decreases from 43 to 20 percent $\left(X_{l m s}=43-20 \%\right)$. Total fraction of limestone is:

$$
X_{l m s}=X_{c a l}+\varphi_{l m s}
$$

Therefore the total fraction of rock is:

$$
X_{\text {rock }}=X_{s s}+X_{l m s}=X_{q t z}+\varphi_{q t z}+X_{c a l}+\varphi_{l m s}=1
$$

Keeping in mind that porosity of limestone is equal to the porosity of sandstone $\left(\varphi_{\text {lms }}=\right.$ $\left.\varphi_{s s}\right)$ and making calculations using above bounding equations, we see that the HashinShtrikman upper and lower bounds are quite tight and are linearly decreasing with increasing porosity. The results are presented on Figure 13. 


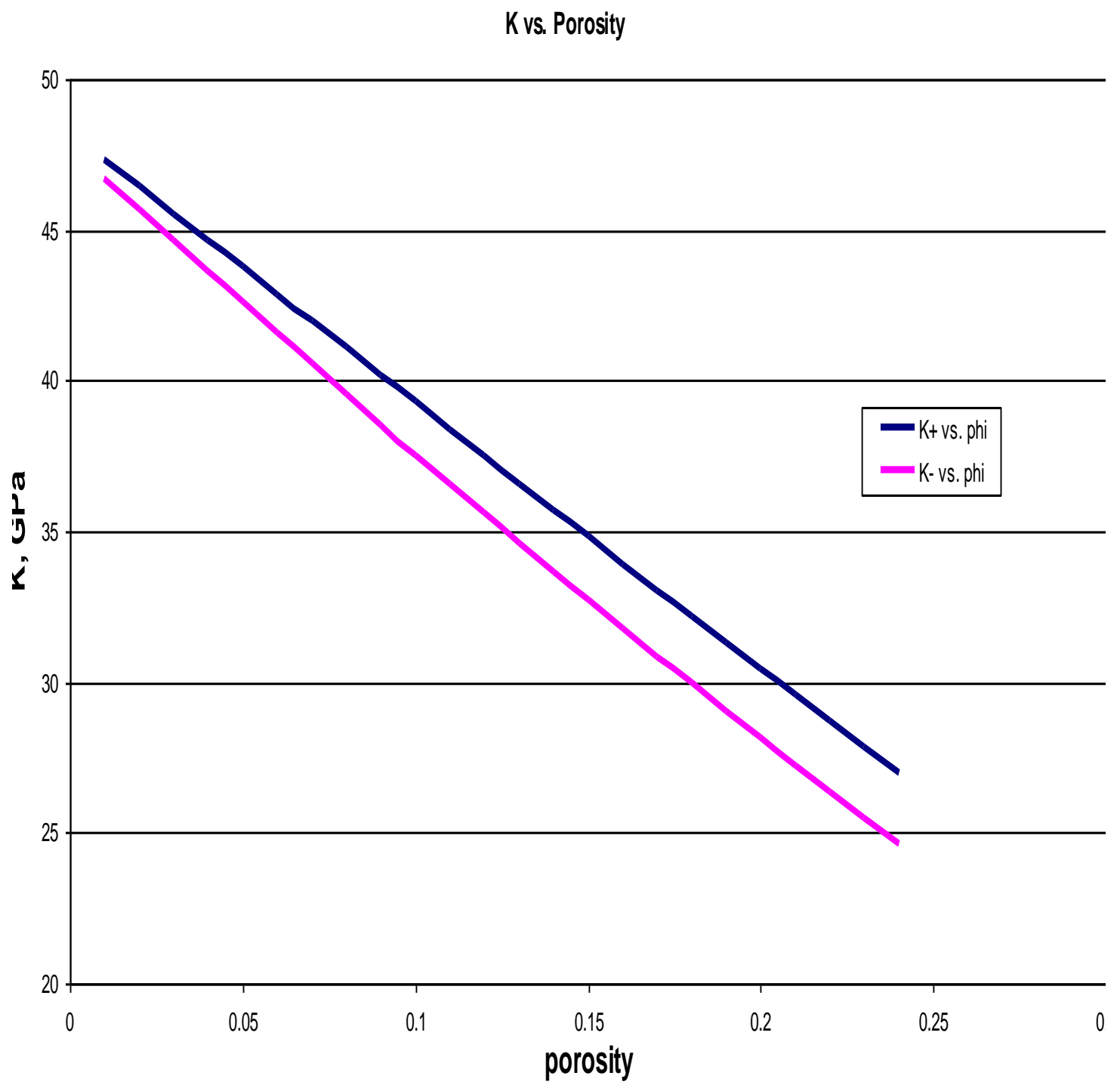

Figure13: Upper (K+) and Lower (K-) Hashin-Shtrikman bounds of multiminerallic rock with equal distribution of porosity between end-members. Porosity of sandstone is equal to porosity of limestone, amount of quartz equals to $56 \%$, amount of calcite changes from $43 \%$ to $20 \%$ as porosity changes from $1 \%$ to $24 \%$. 
To approximate the actual conditions, it is important to take a detailed look into porosity distribution, and consider the case when porosity is apportioned in the most petrologically reasonable way, based on priori information and the best fit of the data. To run calculations of Hashin-Shtrikman bounds we take the same as presented above fraction of end-members in the rock, but porosity now is not equally distributed. Porosity of sandstone varies from 1 to 24 percent $\left(\varphi_{s s}=1-24 \%\right)$, while porosity of limestone is taken to be five percent out of the fraction of limestone:

$$
\varphi_{l m s}=0.05 X_{l m s}
$$

This means that the fraction of calcite within the limestone is:

$$
X_{c a l}=0.95 X_{l m s}
$$

Results of these calculations are presented in Figure 14. This figure shows that with increasing porosity bulk moduli decreases. We know that the shear modulus of calcite $\left(\mu_{c a l}=32 \mathrm{GPa}\right)$ is lower than the shear modulus of quartz $\left(\mu_{q t z}=44 \mathrm{GPa}\right)$, but due to uneven distribution of porosity, the shear modulus of porous limestone is becoming closer to the shear modulus of porous sandstone $\left(\mu_{l m s} \rightarrow \mu_{s s}\right)$. Therefore, comparing Figure 14 with Figure 13, we observe that with the increase in porosity from 0 to 13 percent Hashin-Shtrikman bounds get tighter when $\varphi_{s s} \neq \varphi_{\text {lms }}$ (Figure 14). We also see that upper and lower Hashin-Shtrikman bounds overlie at the point where porosity is around $12-13 \%$, this is because at this point shear moduli of end-members are almost the same $\left(\mu_{s s} \approx \mu_{l m s}\right),($ see Table 2$)$. In case of equal distribution of porosity $\left(\varphi_{s s}=\varphi_{l m s}\right)$ Hashin-Shtrikman bounds get wider with increasing porosity (Figure 13). 


\begin{tabular}{|c|c|c|c|}
\hline Porosity & $\begin{array}{c}(K+)-(K-) \text { equal distribution } \\
\text { of porosity (GPa) }\end{array}$ & $\begin{array}{c}(K+)-(K-) \text { unequal distribution } \\
\text { of porosity (GPa) }\end{array}$ & Equal - Unequal (GPa) \\
\hline 0.01 & 0.612626975 & 0.591300762 & 0.021326214 \\
\hline 0.02 & 0.774126676 & 0.774069588 & 0.000057088 \\
\hline 0.03 & 0.926251444 & 0.909548337 & 0.016703106 \\
\hline 0.04 & 1.06921032 & 0.997393187 & 0.071817133 \\
\hline 0.05 & 1.20321214 & 1.037292457 & 0.165919683 \\
\hline 0.06 & 1.32846551 & 1.028977906 & 0.299487604 \\
\hline 0.07 & 1.445178784 & 0.972236655 & 0.472942129 \\
\hline 0.08 & 1.553560042 & 0.866923637 & 0.686636405 \\
\hline 0.09 & 1.653817058 & 0.71297444 & 0.940842618 \\
\hline 0.1 & 1.74615727 & 0.510418391 & 1.235738879 \\
\hline 0.11 & 1.830787746 & 0.259391696 & 1.571396051 \\
\hline 0.12 & 1.907915144 & 0.039849571 & 1.868065572 \\
\hline 0.13 & 1.977745664 & 0.386916871 & 1.590828793 \\
\hline 0.14 & 2.040485006 & 0.78127726 & 1.259207746 \\
\hline 0.15 & 2.096338303 & 1.222242375 & 0.874095928 \\
\hline 0.16 & 2.145510061 & 1.708958661 & 0.436551401 \\
\hline 0.17 & 2.188204083 & 2.240399125 & -0.052195042 \\
\hline 0.18 & 2.224623378 & 2.815356938 & -0.59073356 \\
\hline 0.19 & 2.254970063 & 3.432441166 & -1.177471103 \\
\hline 0.2 & 2.27944524 & 4.090074923 & -1.810629682 \\
\hline 0.21 & 2.29824886 & 4.78649617 & -2.48824731 \\
\hline 0.22 & 2.311579549 & 5.519761379 & -3.208181829 \\
\hline 0.23 & 2.319634416 & 6.2877522 & -3.968117784 \\
\hline 0.24 & 2.322608806 & 7.088185211 & -4.765576406 \\
\hline
\end{tabular}

Table 2: First column shows fraction of porosity. Second and third columns show differences between upper $\left(\mathrm{K}_{\mathrm{HS}+}\right)$ and lower $\left(\mathrm{K}_{\mathrm{HS}-}\right)$ Hashin-Shtrikman bounds for equal and unequal distributions of porosity respectively. Last column represents difference between values in second and third columns. 


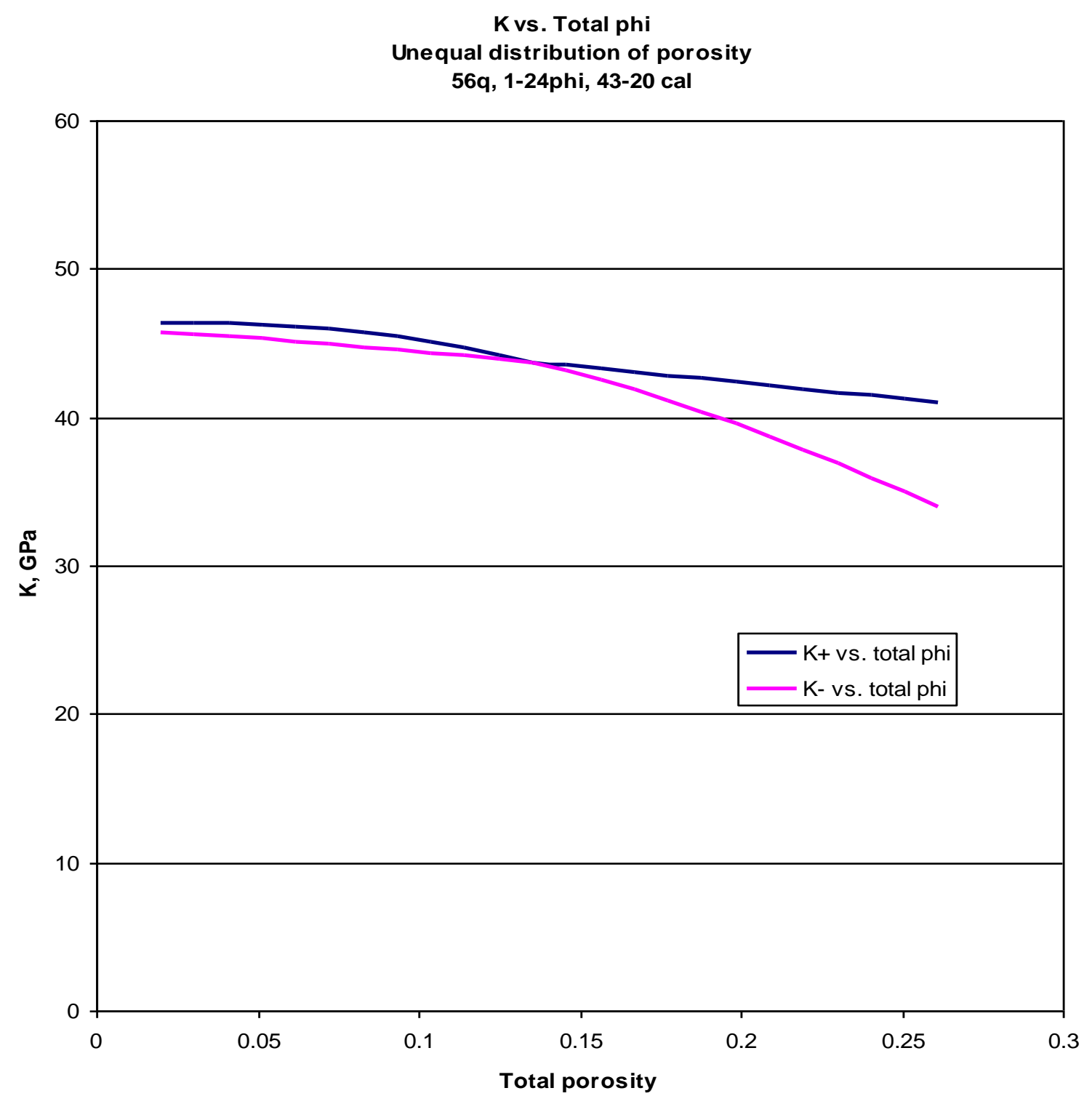

Figure 14. Upper (K+) and Lower (K-) Hashin-Shtrikman bounds of multiminerallic rock with unequal distribution of porosity between end-members. Amount of quartz is $56 \%$. Sandstone porosity changes from 1 to $24 \%$, while limestone porosity changes from 1 to $2.15 \%$ ( $5 \%$ out of total volume of limestone). Calcite changes from 43 to $20 \%$. 
Figures 15-19 represent the values of bulk moduli $(K+$ and $K$-) versus fraction of porosity. In this case we have two changing parameters: porosity of sandstone $\left(\varphi_{s s}\right)$ and fraction of limestone $\left(X_{l m s}\right)$. Porosity of limestone and fraction of quartz are constant:

$$
\varphi_{\text {lms }}=\text { const } ; X_{q t z}=\text { const }
$$

The general behavior of curves is similar to one in Figure 14. At a point where upper $\left(K_{H S+}\right)$ and lower $\left(K_{H S}\right)$ Hashin-Shtrikman bounds overlie, we observe that the shear moduli of end-members are the same:

$$
\mu_{s s}=\mu_{l m s}
$$

Figure 15 to Figure 19 show that as limestone porosity varies, the point where two curves overlie changes. This means that changes in porosity affect Hashin-Shtrikman bounds, i.e. with increase in porosity Hashin-Shtrikman bounds become wider. 
$\mathrm{K}$ vs. sandstone porosity

Upper (K+) and Lower (K-) HS bounds

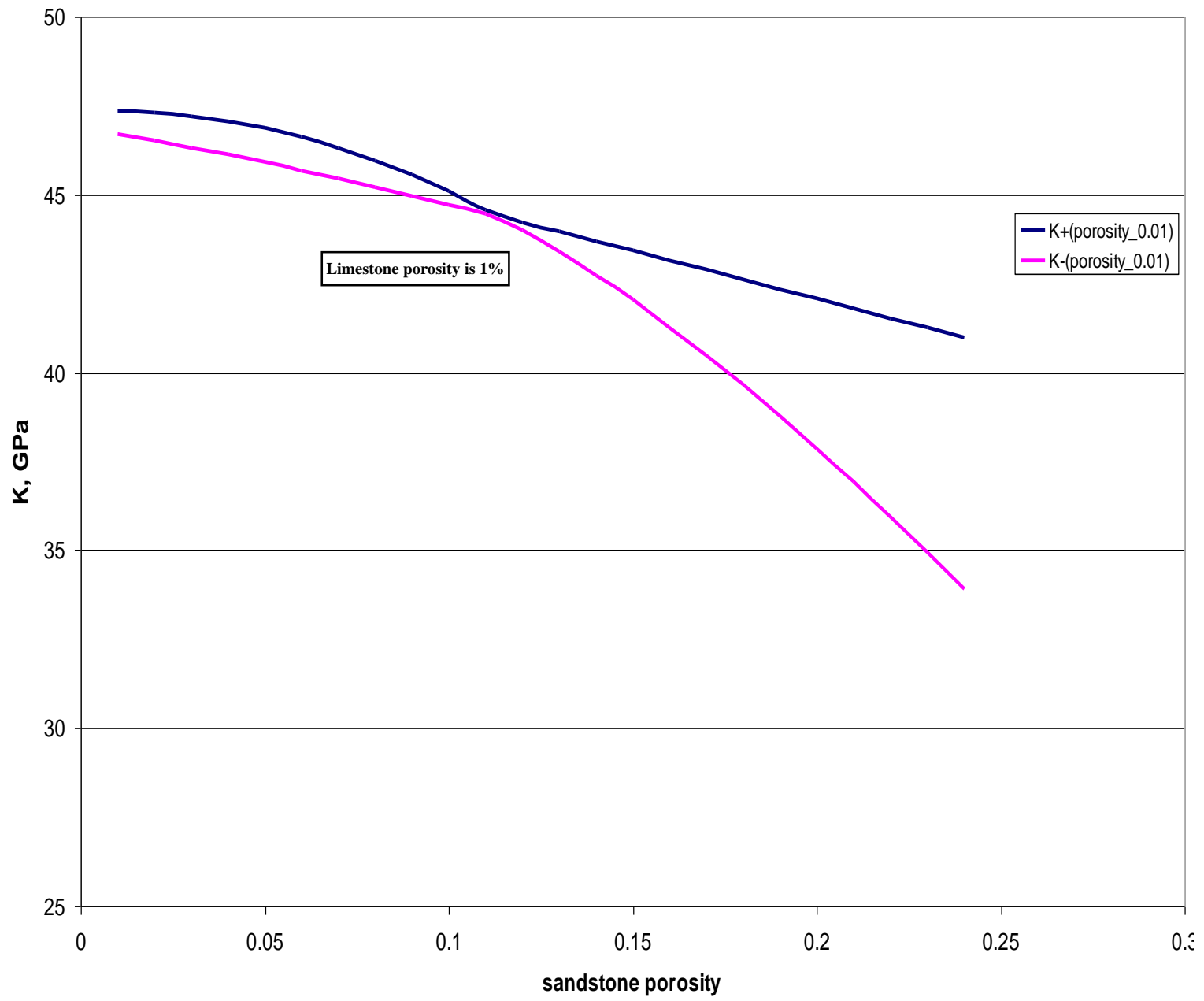

Figure 15: Upper (K+) and Lower (K-) Hashin-Shtrikman bounds of multiminerallic rock with unequal distribution of porosity between end-members. Amount of quartz equals to 56\%. Sandstone porosity changes from 1 to $24 \%$, while limestone porosity stays constant and equals to 1\%. Amount of calcite changes from 43 to $20 \%$. 


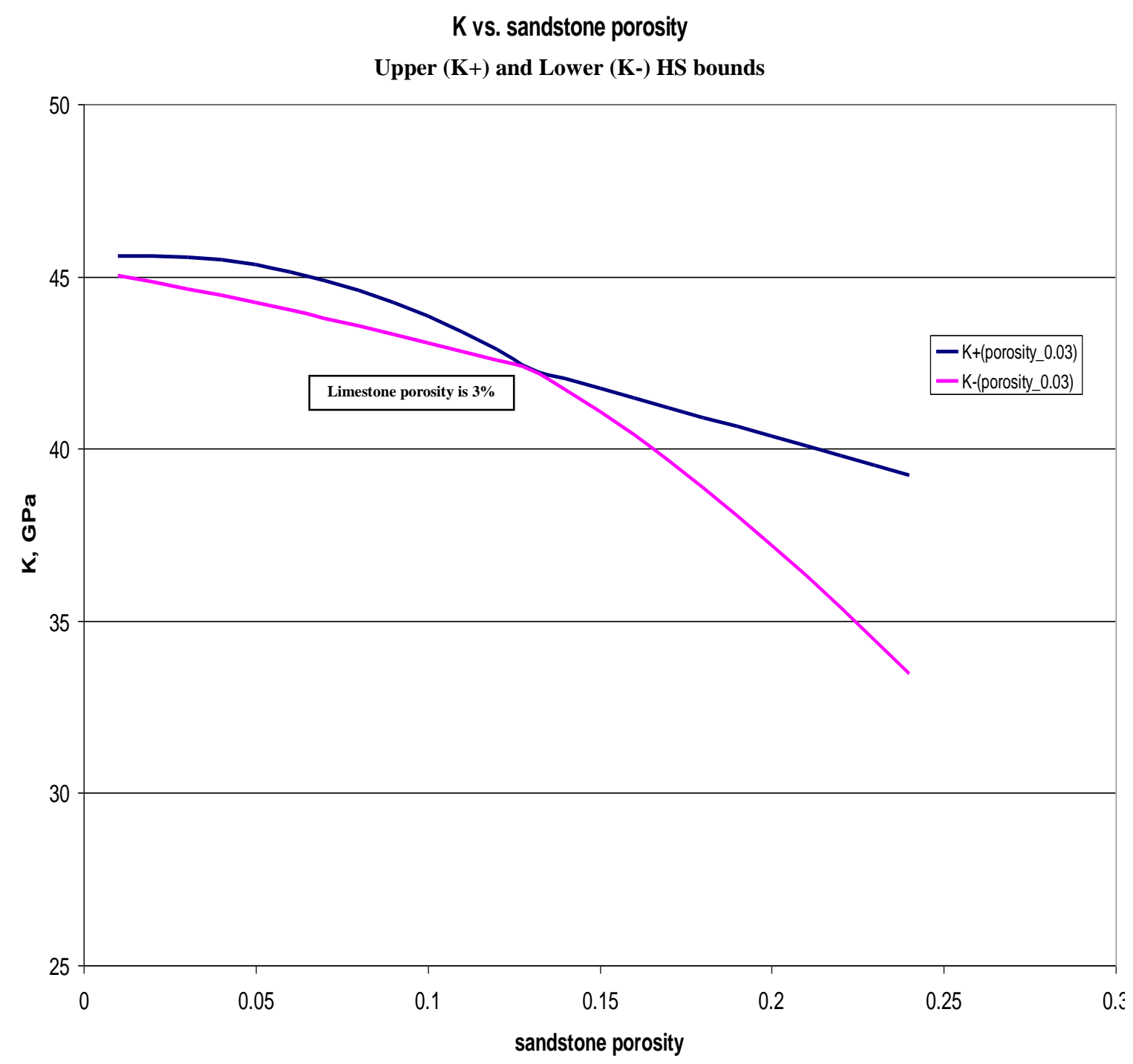

Figure 16: Upper (K+) and Lower (K-) Hashin-Shtrikman bounds of multiminerallic rock with unequal distribution of porosity between end-members. Amount of quartz equals to $56 \%$. Sandstone porosity changes from 1 to $24 \%$, while limestone porosity stays constant and equals to 3\%. Amount of calcite changes from 43 to $20 \%$. 
K vs. sandstone porosity

Upper (K+) and Lower (K-) HS

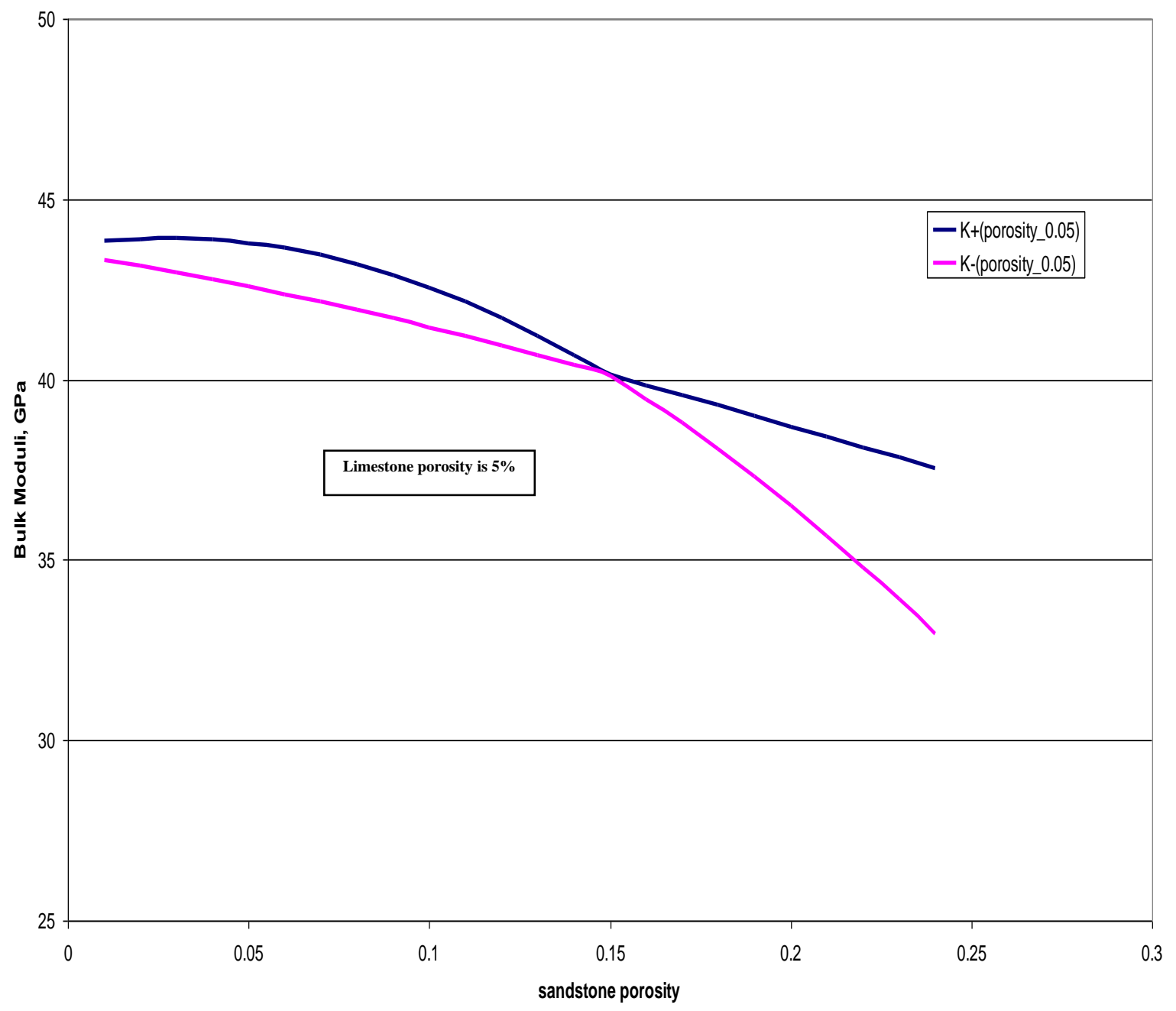

Figure 17: Upper (K+) and Lower (K-) Hashin-Shtrikman bounds of multiminerallic rock with unequal distribution of porosity between end-members. Amount of quartz equals to 56\%. Sandstone porosity changes from 1 to $24 \%$, while limestone porosity stays constant and equals to 5\%. Amount of calcite changes from 43 to $20 \%$ 
K vs. sandstone porosity

Upper (K+) and Lower (K-) HS bounds

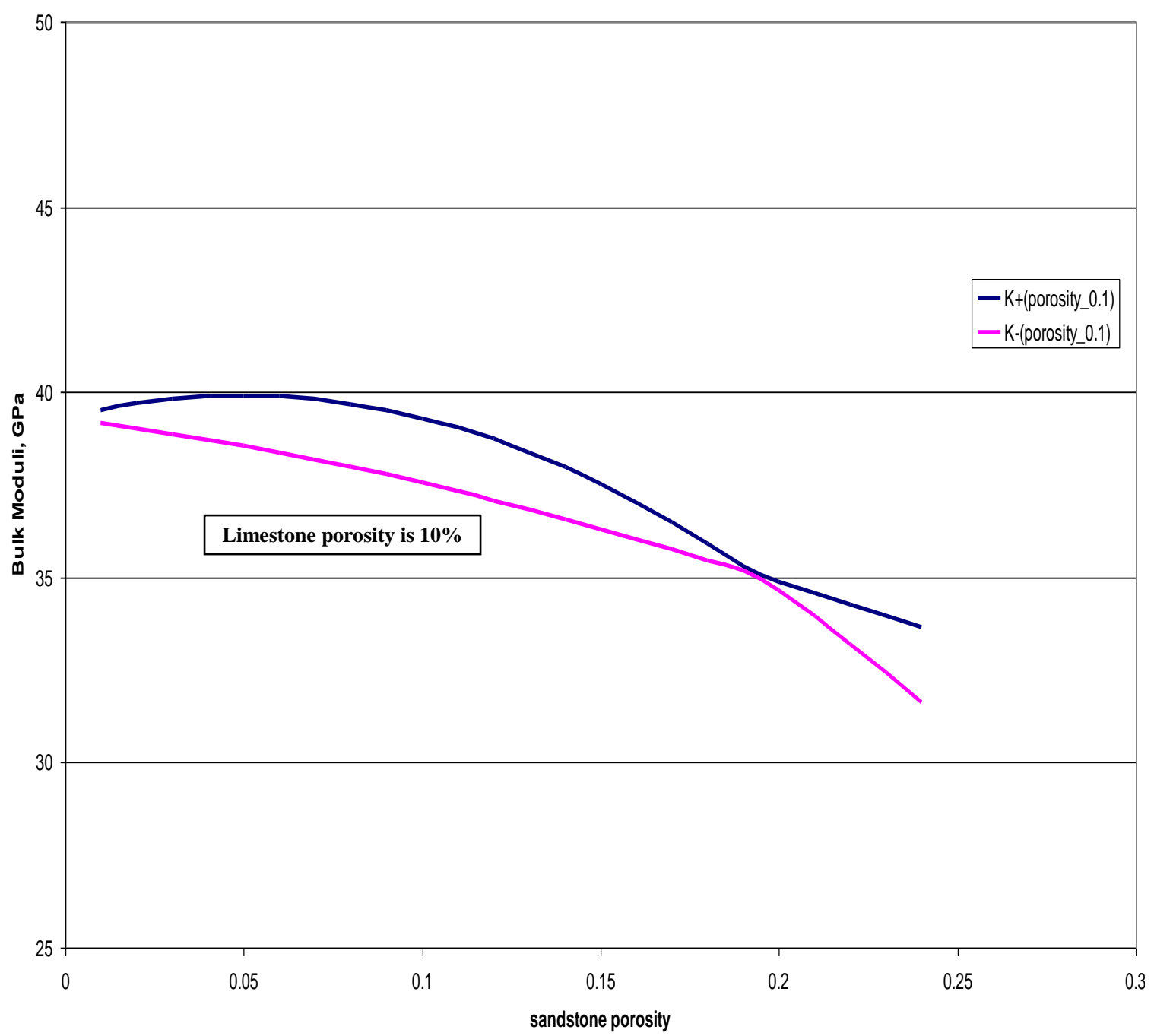

Figure 18: Upper (K+) and Lower (K-) Hashin-Shtrikman bounds of multiminerallic rock with unequal distribution of porosity between end-members. Amount of quartz equals to 56\%. Sandstone porosity changes from 1 to $24 \%$, while limestone porosity stays constant and equals to $10 \%$. Amount of calcite changes from 43 to $20 \%$. 


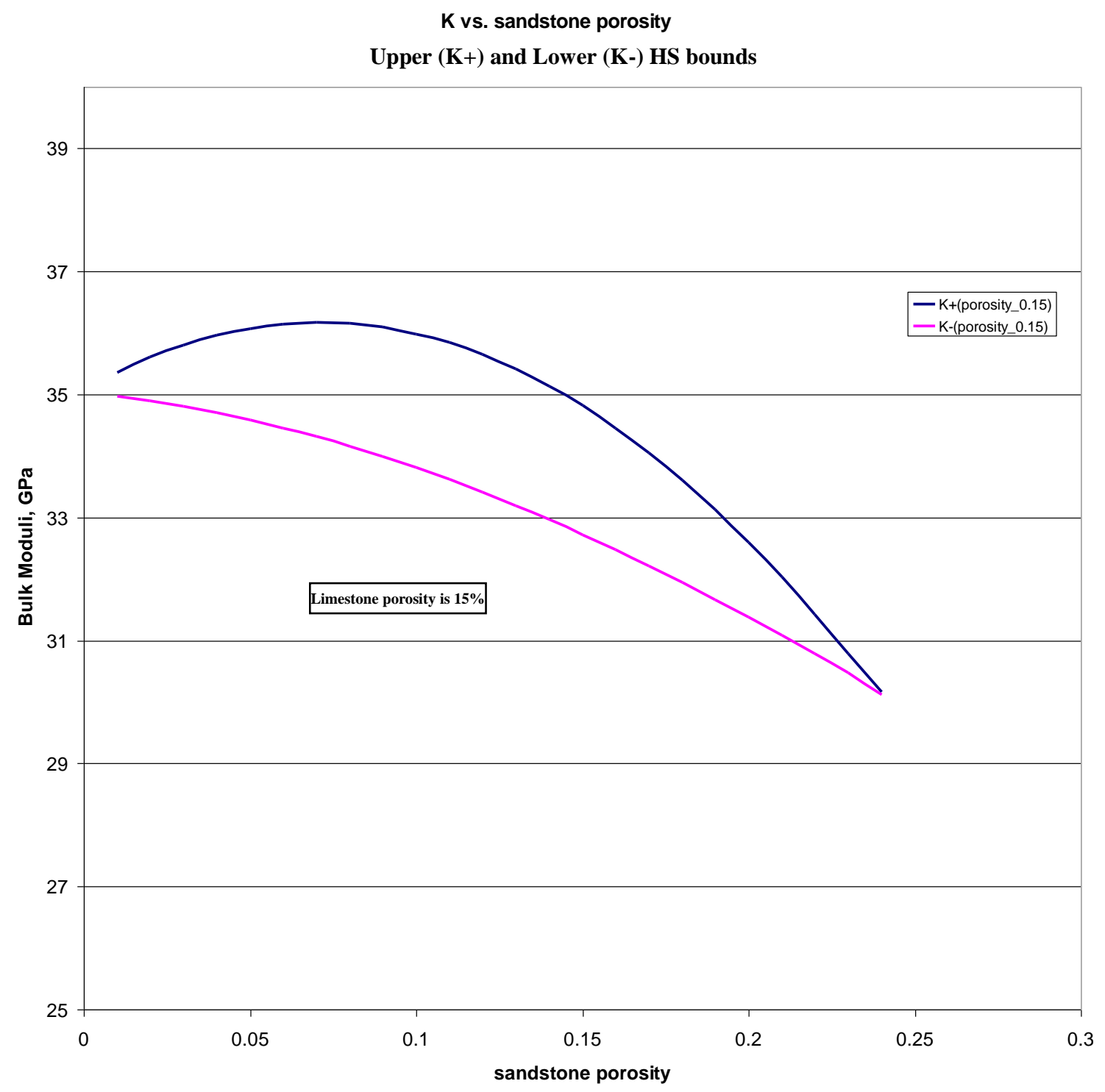

Figure 19: Upper (K+) and Lower (K-) Hashin-Shtrikman bounds of multiminerallic rock with unequal distribution of porosity between end-members. Amount of quartz equals to 56\%. Sandstone porosity changes from 1 to $24 \%$, while limestone porosity stays constant and equals to $15 \%$. Amount of calcite changes from 43 to $20 \%$. 
The explanation of the Hashin-Shtrikman bounds presented on the pictures above lies in examination of elastic moduli of each end-member of the composite. The calculations of bulk moduli of each end-member are presented on Figures 20 and 21 . We see that bulk modulus for sandstone is decreasing dramatically with increasing porosity, while the bulk modulus of limestone is slightly decreasing as porosity of limestone is slightly increasing. Bulk modulus of cement being incorporated into pores of sandstone may have influence on the bulk modulus of the whole rock, but as we see on the figure 21 , porous limestone has bulk modulus changing from 60 to $70 \mathrm{GPa}$, while porous sandstone has bulk modulus below $40 \mathrm{GPa}$. Therefore, it hardly explains why Hashin-Shtrikman upper and lower bounds for rocks with uneven distribution of porosity overlie at some point. 


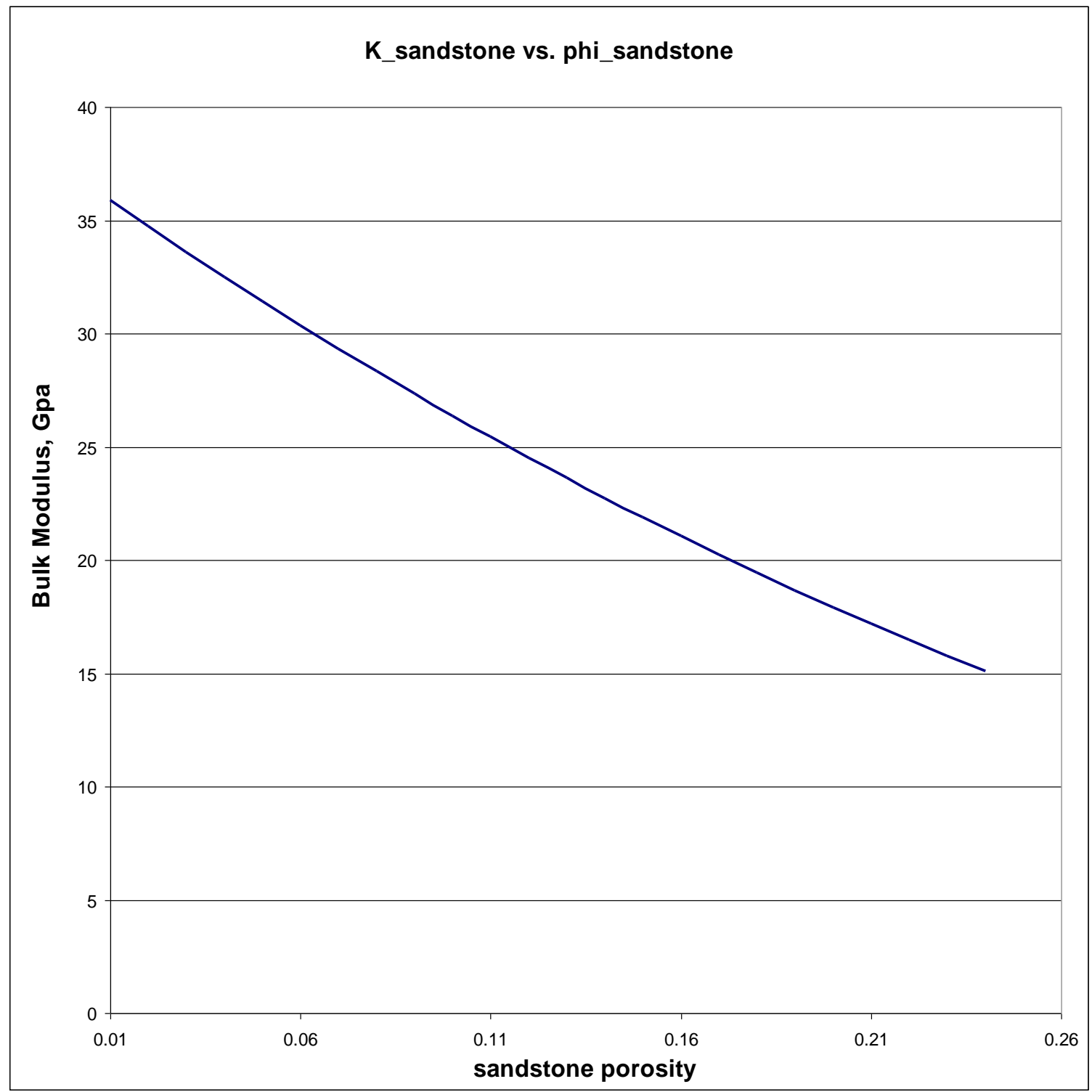

Figure 20: Bulk modulus of sandstone versus porosity of sandstone. With increasing porosity bulk modulus of sandstone is decreasing very rapidly. 


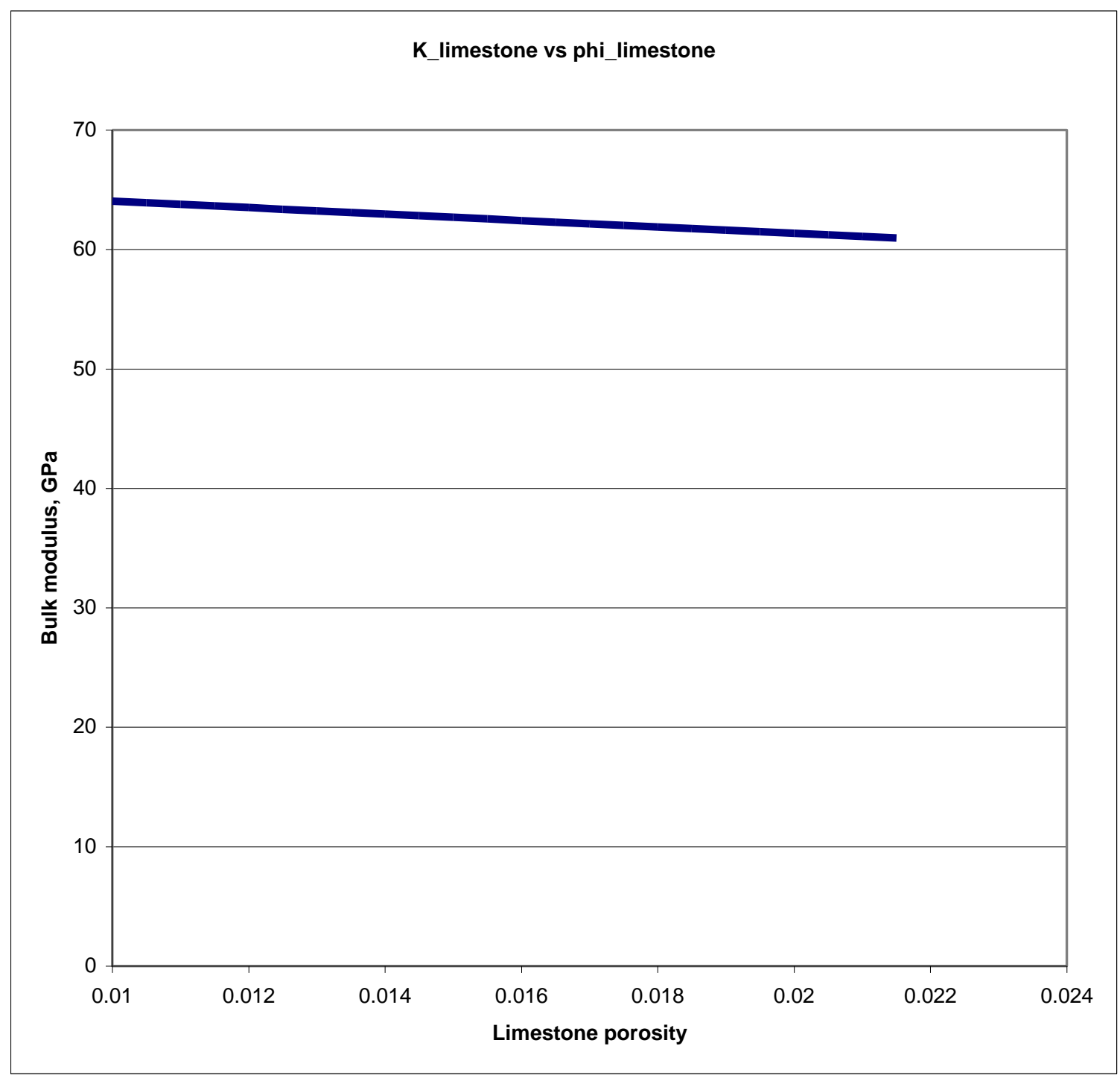

Figure 21: Bulk modulus of limestone versus porosity of limestone. Bulk modulus of limestone is slightly decreasing due to little increase of limestone porosity. 
Results for shear-modulus are presented in Figures 22 and 23. Previously, shear modulus for sandstone decreases fast with decreasing porosity, while the decrease of shear modulus for limestone is slight with slightly decreasing porosity. The general behavior of these curves is similar to the behavior of bulk moduli curves. But, as figure shows, shear modulus for sandstone changes from 10 to $45 \mathrm{GPa}$, while shear modulus of cementing material, which is limestone, changes from 25 to $30 \mathrm{GPa}$. The fact that, shear modulus of both end-members is the same at some points, explains why HashinShtrikman upper and lower bounds overlie. Consequently, at those points where HashinShtrikman bounds overlie we have almost the same shear moduli of porous sandstone and porous limestone. 


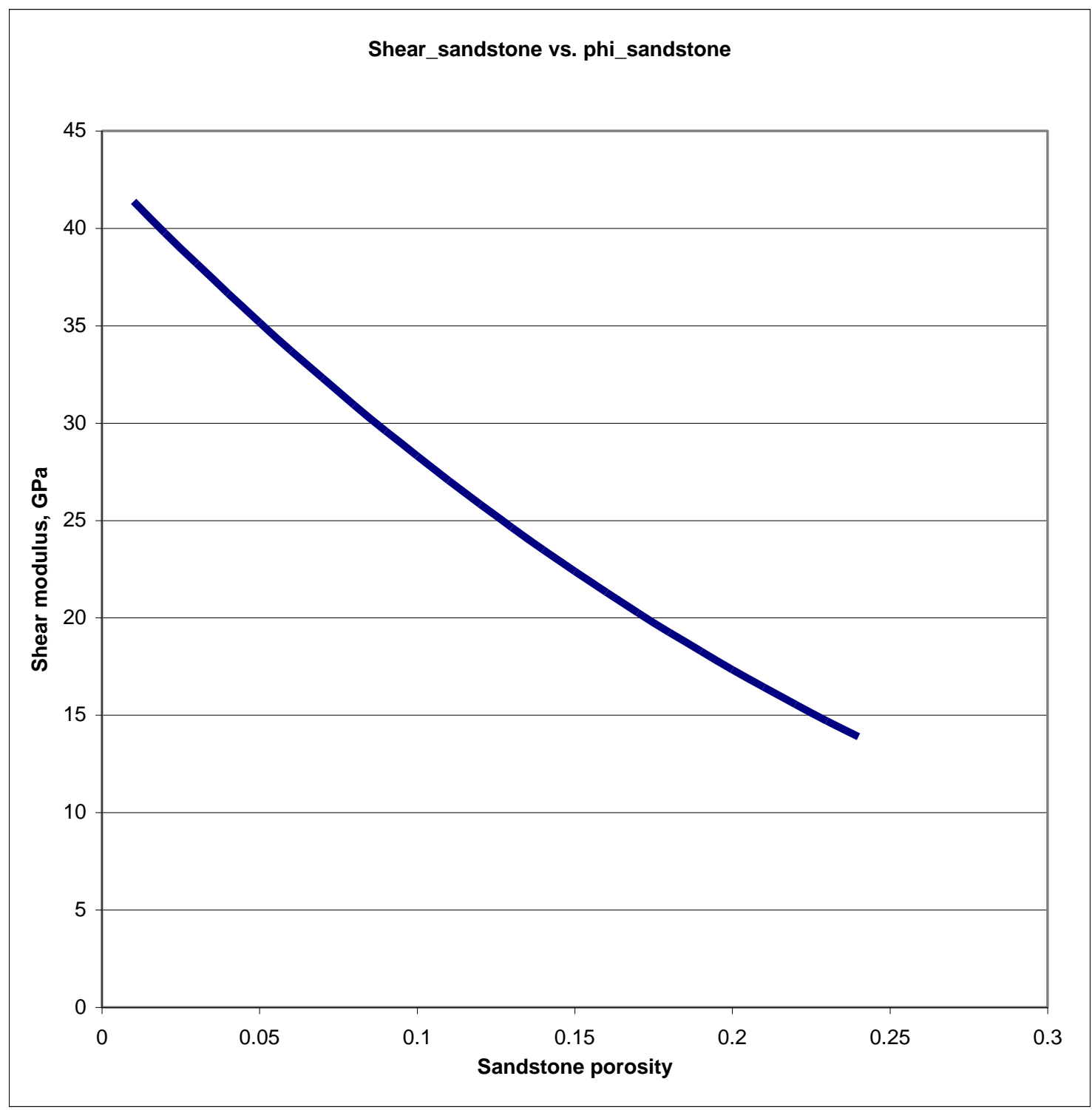

Figure 22. Shear modulus of sandstone versus porosity of sandstone. Shear modulus in this case is abruptly decreasing with increasing porosity. 


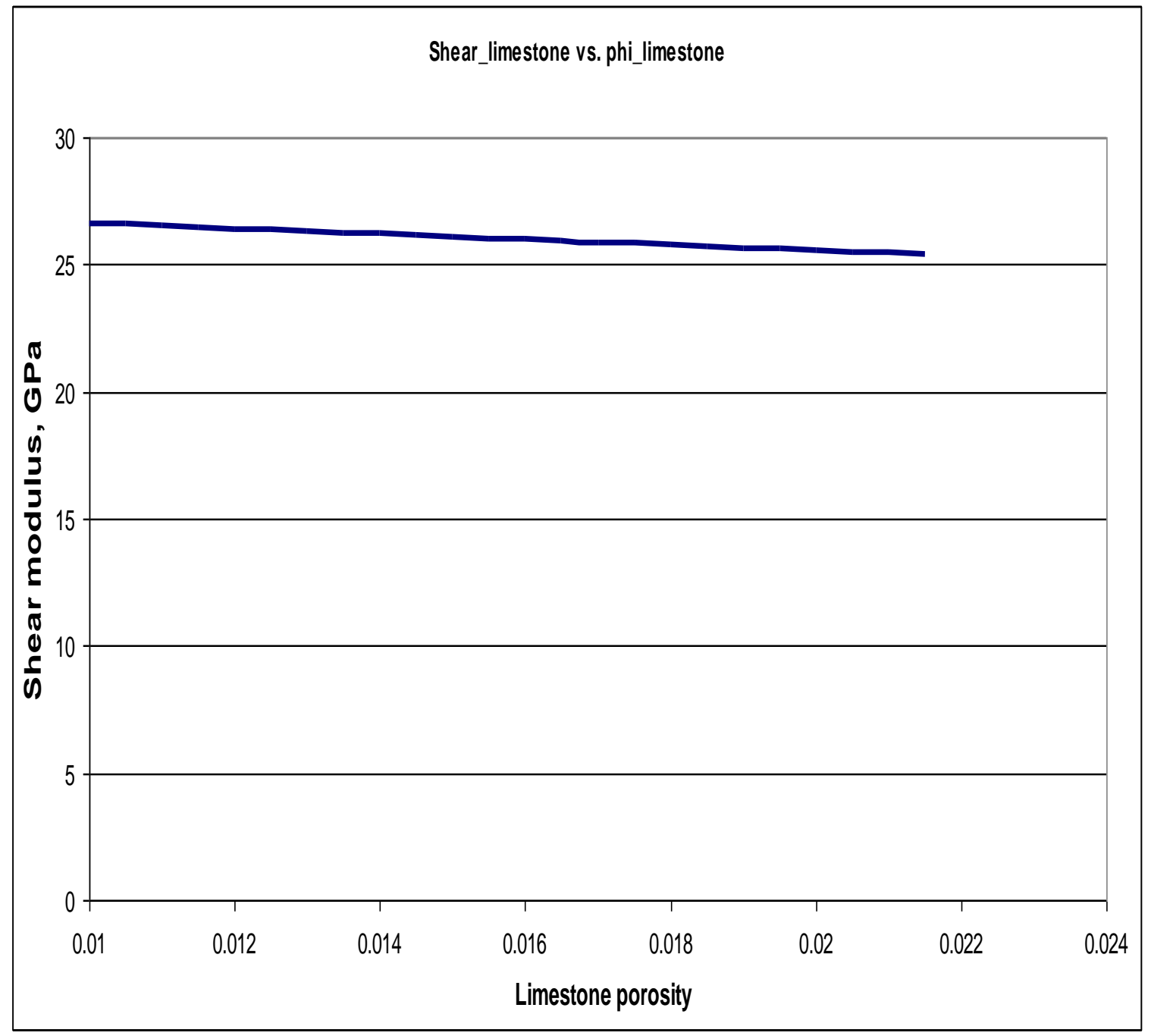

Figure 23: Shear modulus of limestone versus porosity of limestone. Shear modulus decreases very slightly due to little increase of porosity. 


\section{Comparison to Observation}

Figure 24 shows that for the porosity-implicit model, the predicted and observed variations in shear modulus with composition are virtually the same. This fact alone is very significant for shear-wave velocity prediction, and explains why the Greenberg and Castagna (1992) model and others have been successful. However, the theory should explain all the data if correct and the regression fits of Wilkens et al. (1984) show a frame bulk modulus that is more linear with composition than the bounds predict (see Figure 25). What did go wrong? How can the observations violate what theoretically are almost exact bounds? The answer is that the linear regression applied by Wilkens et al., (1984) $a$ priori assumes a linear relationship between velocity and composition. The predicted bounds tell us this cannot be so in the case of $\mathrm{P}$-wave velocity if the assumptions made are correct. However, the porosity-implicit model suggests that a linear velocity versus composition relationship is a very good assumption for shear-wave velocity. In other words, if the theory of Berryman and Milton (1991) is correct, and the porosity is uniformly distributed as assumed, then a linear form of velocity versus composition must be correct for shear waves in the quartz-calcite system, but wrong for $\mathrm{P}-$ waves. The fact that a linear relation is correct for shear-waves may be related to the fact that quartz and calcite shear moduli are more similar than are the bulk moduli. 


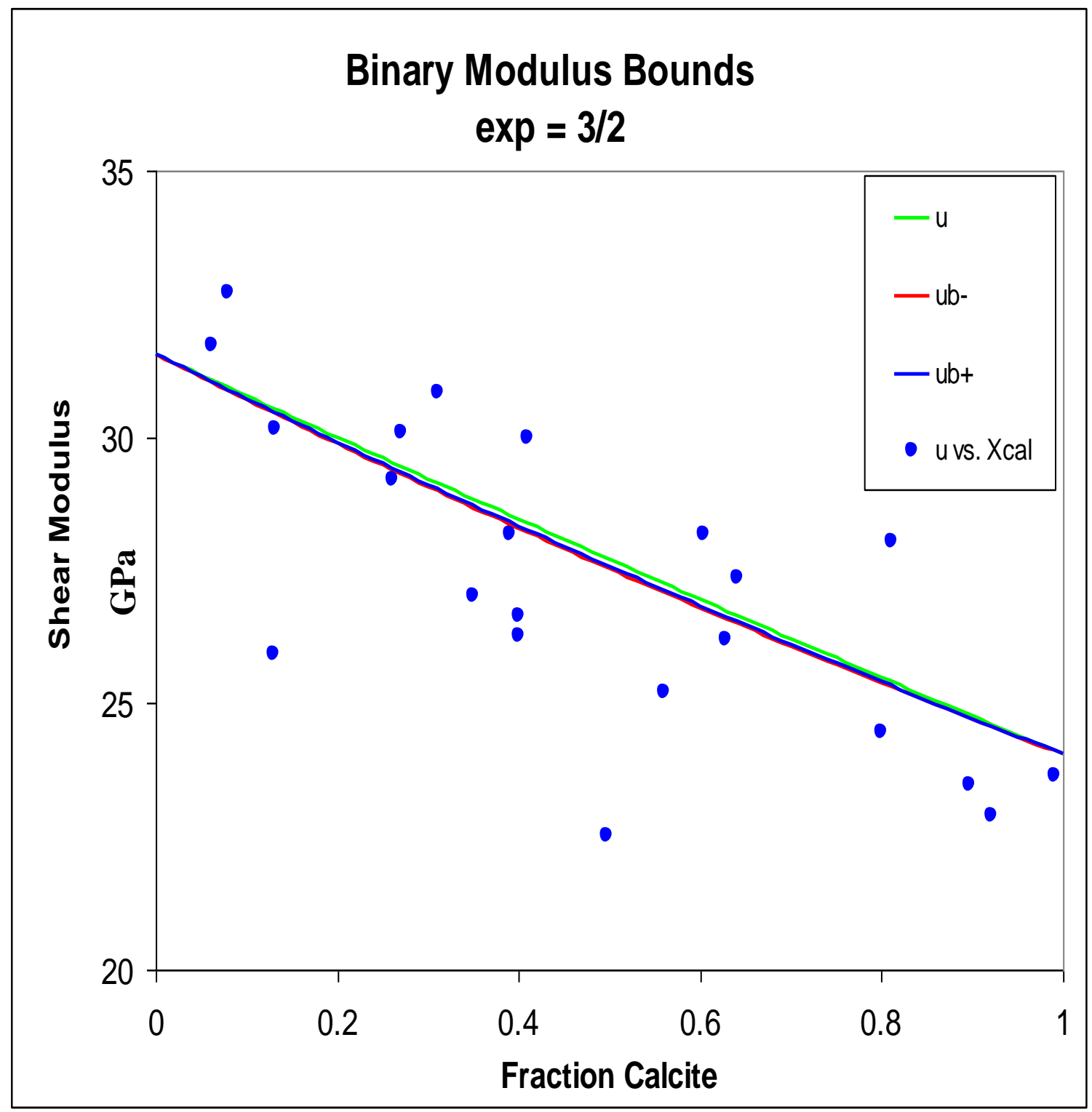

Figure 24. Nearly identical Hashin-Shtrikman upper and lower bounds for shear moduli compared to the linear regression equations of Wilkens (green line) et al. (1984) for porosity equal to 5\%. Hashin - Shtrikman upper and lower bounds are represented by blue and red lines respectively. Points indicate Wilkens data, and each point has different porosity (ranging from 1.3 to $6.8 \%$ ). 
Using the theoretical bounds as a guide, it can be seen that the $\mathrm{P}-$ wave velocity should be related to the $3 / 2$ power of calcite content rather than a simple linear fit (Figure 25). In fact, the $3 / 2$ power equation yields almost identical end-member properties and porosity dependence, fits the theory, and also better fits the data with the same variables as the linear fit (see Table 3).

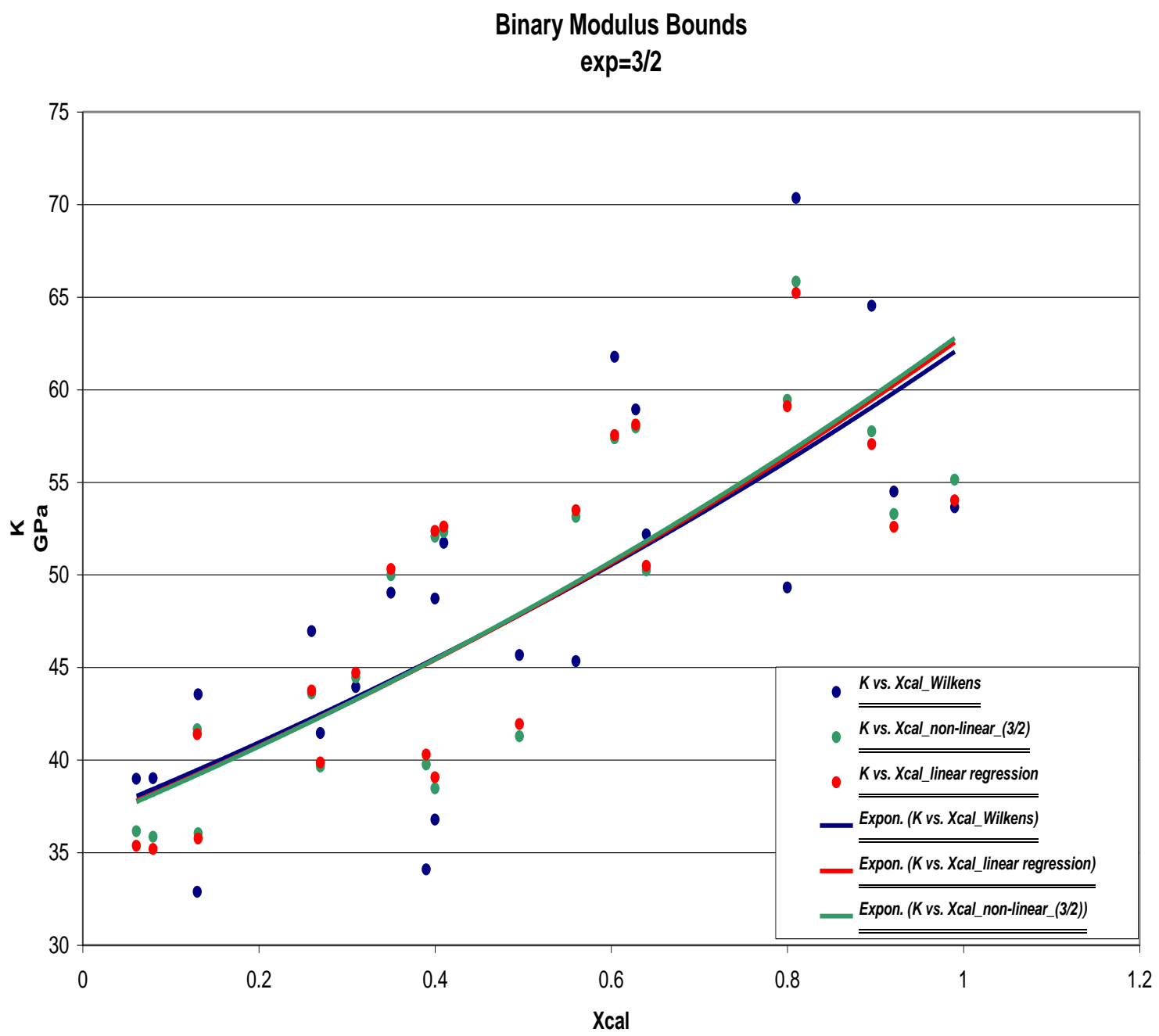

Figure 25: The bulk modulus from linear and non-linear velocity regression fit to the data of the Wilkens et al. (1984). 


\section{Summary of Trends}

\section{- HS Guided Regression}

$$
\begin{aligned}
V_{p}= & 6.08+.419 X_{\text {cal }}^{3 / 2}-13.06 \phi \\
& r=.81 \\
& \text { std err }=.175 \\
& F=16.19 \\
& \text { sig } F=.0000785
\end{aligned}
$$

- Linear Regression

$$
\begin{aligned}
& V_{p}= 6.02+.423 X_{\text {cal }}-12.75 \phi \\
& r=.78 \\
& \text { std err }=.179 \\
& F=14.95 \\
& \text { sig } F=.000126
\end{aligned}
$$

Table 3: Statistical parameters for linear and non-linear regression fit to the data of Wilkens et al, (1984) for measurements at 0.01 kbar. Both regression fits have the same number of variables, yield similar end-member properties and have similar porosity dependence. However, the Hashin-Shtrikman guided non-linear regression fits the data better in a statistically significant manner. 


\begin{tabular}{|c|c|c|c|c|}
\hline Xcal & Porosity & Density & $\begin{array}{c}\text { Dry } \\
\text { Vp_Wilkens }\end{array}$ & K_Wilkens \\
\hline 0.496 & 0.068 & 2.502 & 5.5 & 45.6615 \\
\hline 0.4 & 0.06 & 2.518 & 5.34 & 36.77555787 \\
\hline 0.56 & 0.036 & 2.592 & 5.52 & 45.3371904 \\
\hline 0.4 & 0.024 & 2.54 & 5.76 & 48.719232 \\
\hline 0.81 & 0.013 & 2.672 & 6.35 & 70.3422704 \\
\hline 0.64 & 0.039 & 2.591 & 5.85 & 52.18058083 \\
\hline 0.27 & 0.04 & 2.529 & 5.68 & 41.4563796 \\
\hline 0.131 & 0.047 & 2.533 & 5.75 & 43.5486025 \\
\hline 0.26 & 0.036 & 2.571 & 5.78 & 46.9615432 \\
\hline 0.35 & 0.031 & 2.591 & 5.73 & 49.02785203 \\
\hline 0.08 & 0.039 & 2.552 & 5.69 & 39.01387013 \\
\hline 0.061 & 0.041 & 2.546 & 5.65 & 38.97408313 \\
\hline 0.13 & 0.047 & 2.534 & 5.16 & 32.87172373 \\
\hline 0.921 & 0.056 & 2.563 & 5.76 & 54.48288707 \\
\hline 0.896 & 0.044 & 2.593 & 6.08 & 64.53008947 \\
\hline 0.99 & 0.053 & 2.576 & 5.75 & 53.6356688 \\
\hline 0.8 & 0.034 & 2.613 & 5.6 & 49.3208976 \\
\hline 0.39 & 0.051 & 2.542 & 5.31 & 34.0905078 \\
\hline 0.604 & 0.021 & 2.635 & 6.14 & 61.770724 \\
\hline 0.628 & 0.026 & 2.625 & 5.98 & 58.92145 \\
\hline 0.41 & 0.02 & 2.626 & 5.91 & 51.72055807 \\
\hline 0.31 & 0.031 & 2.59 & 5.73 & 43.933911 \\
\hline
\end{tabular}

Table 4: Wilkens et al, (1984) data plotted on Figure 25 as blue dots. 


\begin{tabular}{|c|c|c|c|c|}
\hline Xcal & Porosity & Density & Vp_calc_linear & K_calc_linear \\
\hline 0.496 & 0.068 & 2.502 & 5.362808 & 41.93279353 \\
\hline 0.4 & 0.06 & 2.518 & 5.4242 & 39.05773619 \\
\hline 0.56 & 0.036 & 2.592 & 5.79788 & 53.48906279 \\
\hline 0.4 & 0.024 & 2.54 & 5.8832 & 52.36271529 \\
\hline 0.81 & 0.013 & 2.672 & 6.19688 & 65.20888207 \\
\hline 0.64 & 0.039 & 2.591 & 5.79347 & 50.47517075 \\
\hline 0.27 & 0.04 & 2.529 & 5.62421 & 39.86143572 \\
\hline 0.131 & 0.047 & 2.533 & 5.476163 & 35.76180893 \\
\hline 0.26 & 0.036 & 2.571 & 5.67098 & 43.75194321 \\
\hline 0.35 & 0.031 & 2.591 & 5.7728 & 50.30345274 \\
\hline 0.08 & 0.039 & 2.552 & 5.55659 & 35.18483001 \\
\hline 0.061 & 0.041 & 2.546 & 5.523053 & 35.3628735 \\
\hline 0.13 & 0.047 & 2.534 & 5.47574 & 41.38122147 \\
\hline 0.921 & 0.056 & 2.563 & 5.695583 & 52.59156148 \\
\hline 0.896 & 0.044 & 2.593 & 5.838008 & 57.05171517 \\
\hline 0.99 & 0.053 & 2.576 & 5.76302 & 54.02180996 \\
\hline 0.8 & 0.034 & 2.613 & 5.9249 & 59.10511935 \\
\hline 0.39 & 0.051 & 2.542 & 5.53472 & 40.28542657 \\
\hline 0.604 & 0.021 & 2.635 & 6.007742 & 57.53723798 \\
\hline 0.628 & 0.026 & 2.625 & 5.954144 & 58.11145578 \\
\hline 0.41 & 0.02 & 2.626 & 5.93843 & 52.60512844 \\
\hline 0.31 & 0.031 & 2.59 & 5.75588 & 44.70380035 \\
\hline
\end{tabular}

Table 5: Data plotted on Figure 25 as red dots; these date show linear regression fit to Wilkens et al (1984) data. 


\begin{tabular}{|c|c|c|c|c|}
\hline Xcal & Porosity & Density & Vp_calc_3/2 & K_calc_3/2 \\
\hline 0.496 & 0.068 & 2.502 & 5.338284764 & 41.27620513 \\
\hline 0.4 & 0.06 & 2.518 & 5.402399547 & 38.46342581 \\
\hline 0.56 & 0.036 & 2.592 & 5.785428498 & 53.11521969 \\
\hline 0.4 & 0.024 & 2.54 & 5.872559547 & 52.04499531 \\
\hline 0.81 & 0.013 & 2.672 & 6.215671 & 65.8321107 \\
\hline 0.64 & 0.039 & 2.591 & 5.785188 & 50.22670824 \\
\hline 0.27 & 0.04 & 2.529 & 5.616384072 & 39.63896445 \\
\hline 0.131 & 0.047 & 2.533 & 5.486046482 & 36.0362463 \\
\hline 0.26 & 0.036 & 2.571 & 5.665388719 & 43.58898081 \\
\hline 0.35 & 0.031 & 2.591 & 5.76189931 & 49.97767029 \\
\hline 0.08 & 0.039 & 2.552 & 5.580140888 & 35.85416831 \\
\hline 0.061 & 0.041 & 2.546 & 5.550852607 & 36.14666018 \\
\hline 0.13 & 0.047 & 2.534 & 5.485819438 & 41.6611939 \\
\hline 0.921 & 0.056 & 2.563 & 5.718982427 & 53.27612415 \\
\hline 0.896 & 0.044 & 2.593 & 5.860726134 & 57.74086561 \\
\hline 0.99 & 0.053 & 2.576 & 5.800550739 & 55.13976654 \\
\hline 0.8 & 0.034 & 2.613 & 5.935771994 & 59.4420635 \\
\hline 0.39 & 0.051 & 2.542 & 5.515989512 & 39.75927024 \\
\hline 0.604 & 0.021 & 2.635 & 6.002424181 & 57.36894608 \\
\hline 0.628 & 0.026 & 2.625 & 5.948962772 & 57.9495649 \\
\hline 0.41 & 0.02 & 2.626 & 5.928799271 & 52.30500273 \\
\hline 0.31 & 0.031 & 2.59 & 5.747459691 & 44.45292862 \\
\hline
\end{tabular}

Table 6: Data presented on Figure 25 as green dots; these date show non-linear regression fit to Wilkens et al (1984) data. 


\section{Conclusions and Discussion}

The Hashin-Shtrikman bounds are an important and useful constraint for frame moduli predictions. In porous rocks, the porosity-implicit approach yields bounds that are sufficiently tight for predictive purposes and mixing models in the quartz-calcite system are linear for shear-wave velocity but non-linear for compressional-wave velocity. The porosity implicit approach can guide non-linear regression fits to the data while limiting the number of variables. The need for empirical trends to consider the compositional dependence of velocity on porosity can be accommodated with the porosity implicit approach. This was not an issue for the highly lithified, low-porosity rocks, studied by Wilkens et al (1984) but is likely to become an issue for assemblages including a wider range of porosities, softer rock frames, clays, and more variable aspect ratio.

Porosity distribution is the key assumption for elastic moduli prediction. In this work results of calculation made on rock assuming equal distribution of porosity were compared with those made on rock of the same mineral composition but possessing unequal distribution of porosity. In case of equal distribution, as porosity changes $\varphi=1-$ $13 \%$, Hashin-Shtrikman upper bound $K_{H S+}$ decreases from around $47.2 \mathrm{GPa}$ to around 36.6 GPa, and Hashin-Shtrikman lower bound $K_{H S-}$ decreases from about $46.7 \mathrm{GPa}$ to about 34.6 GPa. Unequal distribution of porosity shows that with increase of porosity $\varphi$ $=1-13 \%, \mathrm{~K}_{\mathrm{HS}+}$ decreases from $46.6 \mathrm{GPa}$ to $43.6 \mathrm{GPa}$, while $K_{H S}$ - decreases from 45.6 GPa to around $43.5 \mathrm{GPa}$. This shows that if actually we have uneven distribution of 
porosity, but make calculations assuming equal distribution we may have error from around $1.2 \%$ to around $19.1 \%$ for $K_{H S+}$, and from around $2.3 \%$ to around $25.7 \%$ for $K_{H S \text {. }}$

Results also show that if we have unequal distribution of porosity Hashin-Shtrikman bounds are tighter than in case of equal distribution. As shown in Table 2, with increase in porosity from 1 to $13 \%$, Hashin-Shtrikman bounds are tighter from about $0.01 \%$ to around $97.9 \%$. At $\varphi=7 \%, K_{H S}$ bounds calculated assuming uneven distribution of porosity are $32.7 \%$ tighter than $K_{H S}$ bounds calculated assuming even distribution of porosity. For porosities $\varphi>13 \%, K_{H S+}$ and $K_{H S \text { - }}$ become very wide.

The results presented here are theoretically exact for dry rock frames. In the case of saturated rocks, these results are exact only in the limit when permeability approaches zero. The significance of fluid mobility needs to be investigated before these results can be considered applicable for saturated rocks. 


\section{References}

Berryman, J. G., and Milton, G. W., 1991, Exact results for generalized Gassmann's equations in composite porous media with two constituents: Geophysics, 56, 1950-1960

Berryman, J. G., 1995, Mixture theories for rock properties, in A Handbook of Physical Constants, T. J. Ahrens, ed., American Geophysical Union, Washington, D. C., 205228

Castagna, J. P., Batzle, M. L., and Eastwood, R., L., 1985, Relationships between compressional- and shear-wave velocities in clastic silicate rocks: Geophysics, 50, 571581

Castagna, J. P., Batzle, M. L., and Kan, T. K., 1993, Rock physics - the link between rock properties and AVO response: in J. P. Castagna and M. M. Backus eds., OffsetDependent-Reflectivity - Theory and Practice of AVO Analysis, Society of Exploration Geophysicists, Tulsa, 135-171

Gassmann, F., 1951, Elastic waves through a packing of spheres: Geophysics, 16, 673685

Greenberg, M. L., and Castagna, J. P., 1992, Shear-wave velocity estimation in porous rocks: Theoretical formulation, preliminary verification, and applications: Geophys. Prosp., 40, 195-209

Hadley, K., 1976, Comparison of calculated and observed crack densities and seismic velocities in Westerly Granite: J. Geophys. Res., 81, 3484-3494

Han, D., Nur, A., and Morgan, D., 1986, Effects of porosity and clay content on wave velocities in sandstones: Geophysics, 51, 2093-2107 
Hashin, Z., and Shtrikman, S., 1963, A variational approach to the elastic behavior of multiphase materials: J. Mech. Phys. Solids, 11, 127-140

Hill, R., 1952, The elastic behavior of crystalline aggregate: Proc. Physical Soc., London, A65, 349-354

Kuster, G. T., and Toksoz, M. N., 1974, Velocity and attenuation of seismic waves in two-phase media: Geophysics, 39, 587-618

Lamb, W. J., Zhu, Z., McMechan, G. A., Greenberg, M. L., and Castagna, J. P., 1992, Elastic wave propagation in composite media: Geophysics, 57, 1155-1165

Reuss, A., 1929, Berechnung der fliessgrenzen von mischkristallen auf grund der plastizitätsbedingung für einkristalle, Zeitschrift für Angewandie Mathermatik und Mechanik, 9, 49-58

Tosaya, C. A., 1982, Acoustical properties of clay-bearing rocks: Ph. D. Dissertation, Stanford Univ.

Voigt, W., 1928, Lehrbuch der Kristallphysik, p. 962, Teubner, Leipzig

Walpole, L. J., 1966, On bounds for the overall elastic moduli of inhomogeneous systems - 1: J. Mech. Phys. Solids, 14, 151-162

Wilkins, R., Simmons, G., and Caruso, L., 1984, The ratio $\mathrm{V}_{\mathrm{p}} / \mathrm{V}_{\mathrm{s}}$ as a discriminant of composition for siliceous limestones: Geophysics, 49, 1850-1860

Wyllie, M. R. J., Gregory, A. R., and Gardner, L. W., 1956, Elastic wave velocities in heterogeneous and porous media: Geophysics, 21, 41-70

Xu, S., and White, R. E., 1995, A new velocity model for clay-sand mixtures: Geophysical Prospecting: 43, 91-118 